\title{
On near polygons all whose hexes are dual polar spaces
}

\author{
Bart De Bruyn
}

March 26, 2020

\begin{abstract}
One of the most fundamental results in the theory of regular near polygons is the result that every regular near $2 d$-gon, $d \geq 3$, whose parameters $s, t, t_{i}, i \in$ $\{0,1, \ldots, d\}$, satisfy $s, t_{2} \geq 2$ and $t_{3}=t_{2}^{2}+t_{2}$ is a dual polar space. The proof of that theorem heavily relies on Tits' theory of buildings, in particular on Tits' strong results on covering of chamber systems. In this paper, we give an alternative proof which only employs geometrical and algebraic combinatorial arguments.
\end{abstract}

MSC2010: 51A50, 05B25

Keywords: dual polar space, hex

\section{Introduction}

A point-line geometry $\mathcal{S}=(\mathcal{P}, \mathcal{L}, \mathrm{I})$ with nonempty point set $\mathcal{P}$, line set $\mathcal{L}$ and incidence relation $\mathrm{I} \subseteq \mathcal{P} \times \mathcal{L}$ is called a near $2 d$-gon for some $d \in \mathbb{N}$ if the collinearity graph $\Gamma$ of $\mathcal{S}$ has diameter $d$ and if for every point-line pair $(x, L)$, there exists a unique point $\pi_{L}(x)$ on $L$ that is nearest to $x$ with respect to the distance in $\Gamma$. A near 0 -gon is a point and a near 2 -gon is a line. A near quadrangle having a pair of disjoint lines is also called a generalized quadrangle [11]. Every near quadrangle is either a generalized quadrangle or a degenerate generalized quadrangle. The latter is a point-line geometry of diameter 2 that has some distinguished point $x$ such that every point $y \neq x$ is collinear with $x$ and incident with a unique line (containing $x$ ). A near polygon is a near $2 d$-gon for some $d \in \mathbb{N}$. A near polygon is called dense if every line is incident with at least three points and if every two points at distance 2 have at least two common neighbours.

A set $X$ of points of a near polygon $\mathcal{S}$ is called a subspace if every line having two of its points in $X$ has all its points in $X$. Having a nonempty subspace $X$, we denote by $\widetilde{X}$ the subgeometry of $\mathcal{S}$ defined on the point set $X$ by those lines of $\mathcal{S}$ that have all their points in $X$. A set $X$ of points is called convex if every point on a shortest path between two points of $X$ is also contained in $X$. If $X$ is a nonempty convex subspace of a near polygon, then $\widetilde{X}$ clearly is a near polygon. If $\widetilde{X}$ is a generalized quadrangle, then $X$ is called a quad. 
With every polar space $\Pi$ of rank $n \geq 1$ in the sense of Tits [14, Chapter 7], there is associated a dual polar space $\Delta$ of rank $n$. This is the point-line geometry whose points and lines are the maximal and next-to-maximal singular subspaces of $\Pi$, with incidence being reverse containment. By definition, a dual polar space of rank 0 is a point. Every dual polar space of rank $n$ is a near $2 n$-gon. If $F$ is a convex subspace of a dual polar space, then also $\widetilde{F}$ is a dual polar space.

If $x$ and $y$ are two points of a dual polar space or a dense near polygon at distance $i$ from each other, then $x$ and $y$ are contained in a unique convex subspace of diameter $i$. This convex subspace is a quad if $i=2$ and a hex if $i=3$.

The most fundamental characterization result of dual polar spaces (in terms of near polygons) is the following result due to Cameron [6].

Theorem 1.1. The dual polar spaces are precisely the near polygons that satisfy the following two properties:

(1) Every two points at distance 2 are contained in a quad.

(2) For every point-quad pair $(x, Q)$, there is a unique point in $Q$ nearest to $x$.

Theorem 1.1 can be proved in an entirely geometrical way, see [6] and Section 8.3 of [8]. Another characterization result of (finite) dual polar spaces is the following result due to Brouwer and Cohen 3 .

Theorem 1.2. Let $\mathcal{S}$ be a finite near $2 d$-gon, $d \geq 3$, satisfying the following two properties:

(1) Every line of $\mathcal{S}$ has at least three points and every two points at distance 2 have at least three common neighbours (in particular, $\mathcal{S}$ is dense).

(2) If $H$ is a hex, then $\widetilde{H}$ is a dual polar space (of rank 3 ).

Then $\mathcal{S}$ itself is also a dual polar space.

Theorem 1.2 has some applications to regular near polygons. A near polygon is said to have $\operatorname{order}(s, t)$ if every line is incident with precisely $s+1$ points and if every point is incident with exactly $t+1$ lines. A finite near $2 d$-gon with $d \geq 2$ is called regular if there exist constants $s, t, t_{i}, i \in\{0,1, \ldots, d\}$, such that $\mathcal{S}$ has order $(s, t)$ and if $x, y$ are two points at distance $i$ from each other, then $\left|\Gamma_{i-1}(x) \cap \Gamma_{1}(y)\right|=t_{i}+1$. Obviously, $t_{0}=-1$, $t_{1}=0$ and $t_{d}=t$. The collinearity graphs of regular near polygons provide one of the main families of distance-regular graphs, see Chapter 6 of [4].

From Theorem 1.2, the following can be deduced.

Corollary 1.3. If $\mathcal{S}$ is a finite regular near $2 d$-gon, $d \geq 3$, with parameters $s, t, t_{i}, i \in$ $\{0,1, \ldots, d\}$, such that $s, t_{2} \geq 2$ and $t_{3}=t_{2}^{2}+t_{2}$, then $\mathcal{S}$ is a dual polar space. 
Proof. By Theorem 1.2, it suffices to show that $\widetilde{H}$ is a dual polar space for every hex $H$ of $\mathcal{S}$. The hex $H$ is a regular near hexagon with parameters $s^{\prime}, t^{\prime}, t_{i}^{\prime}, i \in\{0,1,2,3\}$, such that $s^{\prime}=s, t^{\prime}=t_{3}$ and $t_{i}^{\prime}=t_{i}$ for every $i \in\{0,1,2,3\}$. The facts that $s^{\prime} \geq 2$ and $t_{2}^{\prime} \geq 1$ imply by [13, Proposition 2.5] that any two points of $\widetilde{H}$ at distance 2 from each other are contained in a quad. Under the assumptions that $s^{\prime} \geq 2$ and $t_{2}^{\prime} \geq 1$, the condition that $t^{\prime}=\left(t_{2}^{\prime}\right)^{2}+t_{2}^{\prime}$ implies by [5. Lemma 25(iii)] that for every point-quad pair $(x, Q)$ of $\widetilde{H}$, there exists a unique point in $Q$ nearest to $x$. Theorem 1.1 then implies that $\widetilde{H}$ is a dual polar space, as we needed to show.

The proof of Theorem 1.2 in [3] heavily relies on Tits' theory of buildings, in particular on Tits' strong results on coverings of chamber systems [15]. As (regular) near polygons are usually studied by means of geometrical and (algebraic) combinatorial techniques, it might therefore be desirable to have a more accessible proof for researchers working in that field. The alternative proof of Theorem 1.2 that will be given here only employs geometrical and algebraical combinatorial techniques and does not rely on the theory of buildings nor on Tits' results on covering of chamber systems. The proof which can be found in Section 5 indeed relies on the theory of distance-regular graphs and on some structural properties (Lemma 4.40) of a family of near polygons that we will derive in Section 4 by entirely geometrical tools.

The author wants to thank Hiroshi Suzuki who expressed his wish to the author to have such a more accessible alternative proof.

\section{Preliminaries}

In this section, we fix some notations and recall some results from the theory of near polygons that will be freely used in Sections 3 and 4 . Most of these results are basic and can be found in the standard references [5], [7, Chapter 1], [8, Chapter 8], [13] on the topics.

Let $\mathcal{S}=(\mathcal{P}, \mathcal{L}, \mathrm{I})$ be a near polygon. If $x$ and $y$ are two points of $\mathcal{S}$, then $\mathrm{d}(x, y)$ denotes the distance between $x$ and $y$ in the collinearity graph $\Gamma$ of $\mathcal{S}$. If $\mathrm{d}(x, y)=1$, then we also write $x \sim y$. If $x$ is a point and $X$ a nonempty set of points, then we define $\mathrm{d}(x, X):=\min \{\mathrm{d}(x, y) \mid y \in X\}$. If $X_{1}, X_{2}$ are two nonempty sets of points, then we define $\mathrm{d}\left(X_{1}, X_{2}\right):=\min \left\{\mathrm{d}\left(x_{1}, x_{2}\right) \mid x_{1} \in X_{1}\right.$ and $\left.x_{2} \in X_{2}\right\}$. If $O$ is a point or a nonempty set of points, then $\Gamma_{i}(O)$ with $i \in \mathbb{N}$ denotes the set of points at distance $i$ from $O$.

Two lines $L_{1}$ and $L_{2}$ of $\mathcal{S}$ at distance $k=\mathrm{d}\left(L_{1}, L_{2}\right)$ from each other are called parallel if $\mathrm{d}\left(x_{1}, L_{2}\right)=\mathrm{d}\left(x_{2}, L_{1}\right)=k$ for every $x_{1} \in L_{1}$ and every $x_{2} \in L_{2}$. If $F$ is a convex subspace of $\mathcal{S}$ and $x \in \Gamma_{1}(F)$, then there exists a unique point $\pi_{F}(x) \in F$ collinear with $x$ and $\mathrm{d}(x, y)=\mathrm{d}\left(x, \pi_{F}(x)\right)+\mathrm{d}\left(\pi_{F}(x), y\right)$ for every $y \in F$. If $F$ is a convex subspace and $x, y$ are two collinear points of $\Gamma_{1}(F)$ such that the line $x y$ does not meet $F$, then $\pi_{F}(x) \sim \pi_{F}(y)$. Moreover, the unique line though $\pi_{F}(x)$ and $\pi_{F}(y)$ coincides with $\pi_{F}(x y):=\left\{\pi_{F}(z) \mid z \in\right.$ $x y\}$ and is parallel with (and at distance 1 from) $x y$.

If $O_{1}, O_{2}, \ldots, O_{k}$ is a collection of $k \geq 1$ points or nonempty sets of points, then $\left\langle O_{1}, O_{2}, \ldots, O_{k}\right\rangle$ denotes the intersection of all convex subspaces containing $O_{1}, O_{2}, \ldots, O_{k}$. 
This is well-defined as $\mathcal{P}$ is such a convex subspace. Note that $\left\langle O_{1}, O_{2}, \ldots, O_{k}\right\rangle$, as intersection of convex subspaces, is again a convex subspace. In fact, it is the smallest convex subspace containing $O_{1}, O_{2}, \ldots, O_{k}$.

Let $\Gamma$ be a graph of diameter $d \geq 2$. $\Gamma$ is called distance-regular if there exist constants $a_{i}, b_{i}, c_{i}(i \in\{0,1, \ldots, d\})$ such that for every two vertices $x$ and $y$ at distance $i \in\{0,1, \ldots, d\}$, we have

$$
\left|\Gamma_{i}(x) \cap \Gamma_{1}(y)\right|=a_{i}, \quad\left|\Gamma_{i+1}(x) \cap \Gamma_{1}(y)\right|=b_{i}, \quad\left|\Gamma_{i-1}(x) \cap \Gamma_{1}(y)\right|=c_{i} .
$$

Obviously, $a_{0}=c_{0}=b_{d}=0, c_{1}=1$ and $\Gamma$ is regular with valency $k=a_{0}+b_{0}+c_{0}=$ $a_{1}+b_{1}+c_{1}=\ldots=a_{d}+b_{d}+c_{d}$.

The regular near $2 d$-gons, $d \geq 2$, are precisely those near $2 d$-gons for which the collinearity graph $\Gamma$ is distance-regular. The connection between the parameters $s, t, t_{i}$ of $\mathcal{S}$ and the parameters $a_{i}, b_{i}, c_{i}$ of $\Gamma$ is given as follows (with $i \in\{0,1, \ldots, d\}$ ):

$$
a_{i}=(s-1)\left(t_{i}+1\right), \quad b_{i}=s\left(t-t_{i}\right), \quad c_{i}=t_{i}+1 .
$$

A polar space of rank $n \geq 1$ in the sense of Tits [14, Chapter 7] is a pair $\Pi=(X, \Sigma)$, where $X$ is a nonempty set whose elements are called points and $\Sigma$ is a set of subsets of $X$, called singular subspaces, for which the following four axioms are satisfied:

(A) A singular subspace together with the singular subspaces contained in it defines a projective space of dimension $r \leq n-1$.

(B) The intersection of any two singular subspaces is again a singular subspace.

(C) If $S$ is an (n-1)-dimensional singular subspace and $x \in X \backslash S$, then there exists a unique $(n-1)$-dimensional singular subspace $S^{\prime}$ containing $x$ and intersecting $S$ in an $(n-2)$-dimensional singular subspace. Moreover, $S \cap S^{\prime}$ consists of those elements of $S$ that are contained with $x$ in a singular subspace of dimension 1 .

(D) There exist two disjoint singular subspaces of dimension $n-1$.

We note that the projective spaces occurring in (A) are allowed to be degenerate (and so can have two points on a line). As mentioned in Section 1, with $\Pi$ there is associated a dual polar space whose points and lines are the $(n-1)$ - and $(n-2)$-dimensional singular subspaces, with incidence being reverse containment. In the case $\Pi$ and $\Delta$ are finite and $\Delta$ satisfies the property that every line is incident with at least three points and every two points at distance 2 have at least three common points, we have the following possibilities for $n \geq 2$ by Tits' classification of polar spaces [14].

\begin{tabular}{|c|c|c|c|c|}
\hline$\Pi$ & $\Delta$ & Ambient space & Defining object & $e$ \\
\hline \hline$W(2 n-1, q)$ & $D W(2 n-1, q)$ & $\mathrm{PG}(2 n-1, q)$ & symplectic polarity & 1 \\
\hline$Q(2 n, q)$ & $D Q(2 n, q)$ & $\mathrm{PG}(2 n, q)$ & parabolic quadric & 1 \\
\hline$Q^{-}(2 n+1, q)$ & $D Q^{-}(2 n+1, q)$ & $\mathrm{PG}(2 n+1, q)$ & elliptic quadric & 2 \\
\hline$H(2 n-1, q), q$ square & $D H(2 n-1, q)$ & $\mathrm{PG}(2 n-1, q)$ & hermitian polarity & $\frac{1}{2}$ \\
\hline$H(2 n, q), q$ square & $D H(2 n, q)$ & $\mathrm{PG}(2 n, q)$ & hermitian polarity & $\frac{3}{2}$ \\
\hline
\end{tabular}


In each case, the polar space $\Pi$ is defined by a polarity $\zeta$ or a quadric $Q$ of the ambient projective space. The singular subspaces are either totally isotropic with respect to $\zeta$ or contained in $Q$. The associated dual polar space $\Delta$ is regular with parameters $s, t, t_{i}$, $i \in\{0,1, \ldots, d\}$, where $s=q^{e}, t_{i}=\frac{q^{i}-1}{q-1}-1(i \in\{0,1, \ldots, d\})$ and $t=t_{d}$.

As the intersection of a quad $Q$ and a convex subspace is a convex subspace, it is either $Q$, a line, a singleton or the empty set. Any two points of a near polygon at distance 2 from each other are thus contained in at most one quad, or equivalently, any two distinct intersecting lines are contained in at most one quad. This implies that the local space $\mathcal{L}_{x}$ in each point is a partial linear space. This local space is defined as the point-line geometry whose points and lines are the lines and quads through $x$, with incident being containment. In a dense near polygon, every two distinct intersecting lines are contained in a unique quad, implying that every local space is a linear space. In a dual polar space $\Delta$ of rank $n$, every local space is a projective space of dimension $n-1$.

A convex subspace $F$ of a near polygon is called classical, if for every point $x$ there exists a unique point $\pi_{F}(x) \in F$ for which $\mathrm{d}(x, y)=\mathrm{d}\left(x, \pi_{F}(x)\right)+\mathrm{d}\left(\pi_{F}(x), y\right)$ for every $y \in F$. Every convex subspace of a dual polar space $\Delta$ is classical.

Two convex subspaces $F_{1}$ and $F_{2}$ of diameter $n-1$ of a near $2 n$-gon are called parallel if each point $x$ of $F_{i}, i \in\{1,2\}$, is collinear with a unique point $\pi_{F_{3-i}}(x)$ of $F_{3-i}$. If this is the case, then $\widetilde{F_{1}} \cong \widetilde{F_{2}}$ by [7, Theorem 1.10]. An isomorphism between $\widetilde{F_{1}}$ and $\widetilde{F_{2}}$ is given by $F_{1} \rightarrow F_{2}: x \mapsto \pi_{F_{2}}(x)$; its inverse is given by $F_{2} \rightarrow F_{1}: x \mapsto \pi_{F_{1}}(x)$.

In the case of a dual polar space $\Delta$ of rank $n$, convex subspaces of diameter $n-1$ are also called maxes. If $M$ is a max of $\Delta$ and $F$ is a convex subspace of diameter $i$ of meeting $M$, then either $F \subseteq M$ or $F \cap M$ is a convex subspace of diameter $i-1$. Two disjoint maxes $M_{1}$ and $M_{2}$ of $\Delta$ are always parallel and every point of $\Delta$ is contained in a quad that intersects $M_{1}$ and $M_{2}$ in lines. For every max $M$ of $\Delta$, there exists a max disjoint from $M$. If $F$ is a convex subspace of diameter $i$ of $\Delta$, then the maximal distance from a point of $\Delta$ to $F$ is equal to $n-i$. In particular, if $M$ is a max, then every point has distance at most 1 from $M$.

If $(x, Q)$ is a point-quad pair of a near polygon $\mathcal{S}$, then at least one of the following cases occurs by [12, Lemma 1.3] (or [7, Theorem 1.22]).

(1) $Q$ contains a unique point $\pi_{Q}(x)$ nearest to $x$. In this case, $\mathrm{d}(x, y)=\mathrm{d}\left(x, \pi_{Q}(x)\right)+$ $\mathrm{d}\left(\pi_{Q}(x), y\right)$ for every $y \in Q$.

(2) The points in $Q$ nearest to $x$ form an ovoid of $Q$, i.e. a set of points of $Q$ meeting each line of the generalized quadrangle $\widetilde{Q}$ in a singleton.

(3) The subgraph of the collinearity graph induced on $Q$ is a bipartite graph and the set of points of $Q$ nearest to $x$ is a proper subset of size at least 2 of one of the two maximal cocliques of this bipartite graph.

The point $x$ is called classical (ovoidal, respectively thin ovoidal) with respect to $Q$ depending on whether case (1), (2) or (3) occurs. 


\section{Quad-closed sets}

In this section, we prove some facts on quad-closed sets of points in dual polar spaces that will be useful in Section 4 .

Suppose $\mathcal{S}=(\mathcal{P}, \mathcal{L}, \mathrm{I})$ is a near polygon with the property that every two points $x_{1}$ and $x_{2}$ at distance 2 are contained in a unique quad, necessarily equal to $\left\langle x_{1}, x_{2}\right\rangle$. A set $X$ of points of $\mathcal{S}$ is called quad-closed if it is a subspace and if for any two points $x_{1}$ and $x_{2}$ of $X$ at distance 2 from each other, the quad $\left\langle x_{1}, x_{2}\right\rangle$ is contained in $X$. Clearly, $\mathcal{P}$ is a quad-closed set and the intersection of any two quad-closed sets is again quad-closed. If $X$ is a set of points of $\mathcal{S}$, then $\langle X\rangle_{q}$ denotes the intersection of all quad-closed sets that contain $X$. Since $\langle X\rangle_{q}$ is also a quad-closed set, it is the smallest quad-closed set that contains the set $X$.

Lemma 3.1. Let $\Delta$ be a dual polar space of rank $n \geq 1$, let $F$ be a max of $\Delta$ and let $L$ be a line of $\Delta$ which intersects $F$ in a point $x$. Then $\langle F \cup L\rangle_{q}$ coincides with the whole set of points of $\Delta$.

Proof. We prove that through every $y \in F$, there exists a line $M_{y}$ which is contained in $\langle F \cup L\rangle_{q}$, but not in $F$. We will prove this by induction on the distance $d(x, y)$. Obviously, the claim is valid if $d(x, y)=0$ since, in this case, we can take $M_{y}$ equal to $L$. Suppose now that $d(x, y)>0$ and let $z \in F$ be a point collinear with $y$ at distance $d(x, y)-1$ from $x$. By the induction hypothesis, there exists a line $M_{z}$ through $z$ which is contained in $\langle F \cup L\rangle_{q}$, but not in $F$. Now, let $M_{y}$ be a line of the quad $\left\langle M_{z}, y z\right\rangle$ through $y$ distinct from $y z$. Since $M_{z} \cup y z \subseteq\langle F \cup L\rangle_{q}$, the quad $\left\langle M_{z}, y z\right\rangle$ is completely contained in $\langle F \cup L\rangle_{q}$. In particular, $M_{y}$ is contained in $\langle F \cup L\rangle_{q}$. Since $y z=\left\langle M_{z}, y z\right\rangle \cap F$ and $M_{y} \neq y z, M_{y}$ is not contained in $F$.

Now, let $u$ be an arbitrary point of $\Delta$. If $u \in F$, then $u \in\langle F \cup L\rangle_{q}$. Suppose therefore that $u \notin F$, let $v$ be the unique point of $F$ collinear with $u$ and let $L_{v}$ denote a line through $v$ contained in $\langle F \cup L\rangle_{q}$, but not in $F$. Such a line exists by the previous paragraph. If $u \in L_{v}$, then $u \in\langle F \cup L\rangle_{q}$. If $u \notin L_{v}$, then the quad $\left\langle u v, L_{v}\right\rangle$ intersects $F$ in a line $M$ and we have $\left\langle u v, L_{v}\right\rangle=\left\langle L_{v}, M\right\rangle$. Since $L_{v} \cup M \subseteq\langle F \cup L\rangle_{q}$, we have $\left\langle u v, L_{v}\right\rangle \subseteq\left\langle L_{v}, M\right\rangle \subseteq\langle F \cup L\rangle_{q}$. In particular, $u \in\langle F \cup L\rangle_{q}$.

We can generalize Lemma 3.1 as follows.

Lemma 3.2. Let $\Delta$ be a dual polar space of rank $n \geq 1$, let $X$ be a nonempty subspace of $\Delta$ such that $\widetilde{X}$ is connected. Then $\langle X\rangle_{q}=\langle X\rangle$.

Proof. Let $k$ be the diameter of $\langle X\rangle$. We construct by induction a chain $F_{0} \subset F_{1} \subset$ $\cdots \subset F_{k}$ of convex subspaces satisfying: (i) $F_{i}, i \in\{0,1, \ldots, k\}$, has diameter $i$; (ii) $F_{i}$, $i \in\{0,1, \ldots, k\}$, is contained in $\langle X\rangle$. These conditions imply that $F_{k}=\langle X\rangle$.

Let $F_{0}=\left\{y_{0}\right\}$ be any singleton contained in $X$. Suppose that we have defined $F_{i}$ for a certain $i \in\{0, \ldots, k-1\}$. Then $F_{i}$ has diameter $i<k$ and is properly contained in $\langle X\rangle$. So, by the connectedness of $\tilde{X}$, there is a point $y_{i} \in X \backslash F_{i}$ which is collinear with some 
point $z_{i}$ of $F_{i}$. Then we define $F_{i+1}=\left\langle F_{i}, z_{i} y_{i}\right\rangle=\left\langle F_{i}, y_{i}\right\rangle$. Clearly, $F_{i+1}$ has diameter $i+1$ and is contained in $\langle X\rangle$.

Using the just-constructed chain $F_{0} \subset F_{1} \subset \cdots \subset F_{k}$, we show that $\langle X\rangle_{q}=\langle X\rangle$. Obviously, we have $\langle X\rangle_{q} \subseteq\langle X\rangle=F_{k}$. In order to prove that $\langle X\rangle_{q}=\langle X\rangle$, it suffices to prove by induction on $i \in\{0,1, \ldots, k\}$ that $F_{i} \subseteq\langle X\rangle_{q}$. Obviously, this is true if $i=0$ (since $F_{0}=\left\{y_{0}\right\} \subseteq X$ ) and if $i=1$ (since $F_{1}=y_{0} y_{1} \subseteq X$ ). So, suppose that $i \in\{1,2, \ldots, k-1\}$ and that $F_{i}$ is contained in $\langle X\rangle_{q}$. Let $L_{i}$ be the line $y_{i} z_{i}$. Since $F_{i} \cup\left\{y_{i}\right\} \subseteq\langle X\rangle_{q}$, we have $F_{i} \cup L_{i} \subseteq\langle X\rangle_{q}$. By Lemma 3.1. $\left\langle F_{i}, L_{i}\right\rangle_{q}=\left\langle F_{i}, L_{i}\right\rangle=F_{i+1}$. Since $F_{i} \cup L_{i} \subseteq\langle X\rangle_{q}$, we necessarily have that $F_{i+1}=\left\langle F_{i}, L_{i}\right\rangle_{q} \subseteq\langle X\rangle_{q}$.

\section{A family of near polygons}

In this section, we study in an entirely geometrical way a family of near polygons that include the dense near polygons and the dual polar spaces. One of the results we obtain here (Lemma 4.40) will play an essential role in the proof of Theorem 1.2 that will be given in Section 5 .

Let $\mathcal{S}=(\mathcal{P}, \mathcal{L}, \mathrm{I})$ be a near $2 n$-gon, $n \geq 4$, that satisfies the following three properties:

(P1) For any two points $x$ and $y$ of $\mathcal{S}$, the convex subspace $\langle x, y\rangle$ has diameter $\mathrm{d}(x, y)$.

(P2) Every two points at distance 2 have at least two common neighbours.

(P3) If $M$ is a max of $\mathcal{S}$, then $\widetilde{M}$ is a dual polar space of rank $n-1$.

For near polygons satisfying properties (P1) and (P2), a max is defined as a convex subspace of diameter $n-1$, and a hex is defined as a convex subspace of diameter 3 . Every dense near polygon and every dual polar space satisfies properties (P1) and (P2), see e.g. Sections 6.9 and 8.1 of [8].

Our ultimate goal here will be to show that $\mathcal{S}$ has special subgeometries that are dual polar spaces of rank $n$, and to determine several properties of these subgeometries. These goals will be achieved in the final lemmas and theorems of this section. First, we need to do some preparatory work.

The first fourteen results that we will derive here (Lemma 4.1 till Lemma 4.14) are valid for general near polygons satisfying properties (P1) and (P2) (without requiring (P3)).

Lemma 4.1. Every two points at distance 2 are contained in a (necessarily unique) quad.

Proof. Let $x$ and $y$ be two arbitrary points at distance 2 from each other, and put $Q:=$ $\langle x, y\rangle$. By property (P1), $Q$ has diameter 2 and so the near quadrangle $\widetilde{Q}$ is either a generalized quadrangle or a degenerate generalized quadrangle. The last possibility cannot occur as otherwise $x$ and $y$ have a unique neighbour, which is in contradiction with property (P2). So, $\widetilde{Q}$ is a generalized quadrangle and $Q$ is a quad. 
Lemma 4.2. (1) Every two distinct intersecting lines are contained in a unique quad. As a consequence, every local space is linear.

(2) Any two parallel lines at distance 1 from each other are contained in a unique quad.

Proof. (1) If $L_{1}$ and $L_{2}$ are two distinct intersecting lines and $x_{i}$ with $i \in\{1,2\}$ is a point of $L_{i}$ not contained in $L_{3-i}$, then the quads containing $L_{1}$ and $L_{2}$ are precisely the quads containing $x_{1}$ and $x_{2}$, and so there is a unique such quad by Lemma 4.1 .

(2) Let $L_{1}$ and $L_{2}$ be two parallel lines at distance 1 from each other and let $L_{3}$ and $L_{4}$ be two distinct lines meeting $L_{1}$ and $L_{2}$ in points. Any quad containing $L_{1}$ and $L_{2}$ also contains $L_{3}$ and so must coincide with the quad $\left\langle L_{1}, L_{3}\right\rangle$.

It remains to show that the quad $Q=\left\langle L_{1}, L_{3}\right\rangle$ also contains the line $L_{2}$. As the point $L_{2} \cap L_{4}$ is collinear with the points $L_{2} \cap L_{3}$ and $L_{1} \cap L_{4}$ of $Q$, it is contained in $Q$, implying that also the lines $L_{2}$ and $L_{4}$ are contained in $Q$.

Lemma 4.3. Every point-quad pair is classical or ovoidal.

Proof. Suppose $Q$ is a quad and $x$ is a point that is thin-ovoidal with respect to $Q$. Then $\widetilde{Q}$ is a dual grid. Put $\mathrm{d}(x, Q)=k$. Then $\left|\Gamma_{k}(x) \cap Q\right| \geq 2, \Gamma_{k+2}(x) \cap Q \neq \emptyset$ and $C_{1}:=\left(\Gamma_{k}(x) \cap Q\right) \cup\left(\Gamma_{k+2}(x) \cap Q\right)$ and $C_{2}:=\Gamma_{k+1}(x) \cap Q$ are the two maximal cocliques of $Q$.

Let $y \in \Gamma_{k+1}(x) \cap Q$, and consider the convex subspace $\langle x, y\rangle$ of diameter $k+1$. Every point of $\Gamma_{k}(x) \cap Q$ is collinear with $y$ and so is contained in the convex subspace $\langle x, y\rangle$. As $\left|\Gamma_{k}(x) \cap Q\right| \geq 2$ and $\Gamma_{k}(x) \cap Q$ is a coclique, this implies that the whole quad $Q$ is contained in the convex subspace $\langle x, y\rangle$. So, the convex subspace $\langle x, y\rangle$ of diameter $k+1$ contains points at distance $k+2$ from $x$ (namely the points of $\Gamma_{k+2}(x) \cap Q$ ), which is impossible.

Lemma 4.4. If $F$ is a convex subspace of diameter $\delta$ of $\mathcal{S}$, then every shortest path of length $i<\delta$ in $F$ can be extended to a shortest path of length $\delta$ in $F$.

Proof. Consider a shortest path of length $i$ between two points $x$ and $y$ of $F$. Let $F^{\prime}$ denote the convex subspace $\langle x, y\rangle$ of diameter $i$. As $F$ is a convex subspace, we have $F^{\prime}=\langle x, y\rangle \subseteq F$. As $i<\delta$, there exists a line $L \subseteq F$ intersecting $F^{\prime}$ in a singleton $\{z\}$. We suppose that we have chosen the line $L$ in such a way that $\mathrm{d}(y, z)$ is minimal. We show that $z=y$.

Suppose $z \neq y$, and let $z^{\prime}$ be a point of $\Gamma_{1}(z)$ at distance $\mathrm{d}(z, y)-1$ from $y$. As $F^{\prime}$ is a convex subspace, we have $z z^{\prime} \subseteq F^{\prime}$. As $L$ is not contained in $F^{\prime}$, the quad $Q=\left\langle L, z z^{\prime}\right\rangle$ intersects $F^{\prime}$ in $z z^{\prime}$. So, any line $U$ of $Q$ through $z^{\prime}$ distinct from $z z^{\prime}$ would be contained in $F$ (as $L, z z^{\prime} \subseteq F$ and $F$ is convex) and intersect $F^{\prime}$ in $z^{\prime}$, in contradiction with the minimality of $\mathrm{d}(y, z)$.

So, we have $z=y$. If $u \in L \backslash\{y\}$, then $\mathrm{d}\left(u, F^{\prime}\right)=1$ and $y=\pi_{F^{\prime}}(u)$. So, $\mathrm{d}(u, x)=$ $\mathrm{d}(u, y)+\mathrm{d}(y, x)=i+1$. We can thus extend the path to a path of length $i+1$ in $F$. A straightforward inductive argument then shows that the path can be extended to a shortest path of length $\delta$ in $F$. 
The following is an immediate consequence of Lemma 4.4 .

Corollary 4.5. If $F$ is a convex subspace of diameter $\delta$, then for every point $x \in F$, there exists a point $y \in F$ at distance $\delta$ from $x$.

Lemma 4.6. Every two points at distance $\delta$ are contained in a unique convex subspace of diameter $\delta$.

Proof. Let $x$ and $y$ be two points at distance $\delta$ from each other. By property (P1), $\langle x, y\rangle$ is a convex subspace of diameter $\delta$ containing $x$ and $y$. Suppose $F \neq\langle x, y\rangle$ is another convex subspace of diameter $\delta$ containing $x$ and $y$. As $\langle x, y\rangle$ is the smallest convex subspace containing $x$ and $y,\langle x, y\rangle$ is properly contained in $F$. As $F$ is connected, there exists some point $u \in F \backslash\langle x, y\rangle$ that is collinear with a point $v \in\langle x, y\rangle$. By Corollary 4.5, we know that there exists some point $w \in\langle x, y\rangle$ at distance $\delta$ from $v$. Now, $\mathrm{d}(u,\langle x, y\rangle)=1$ and $v$ is the unique point of $\langle x, y\rangle$ collinear with $u$, implying that $\mathrm{d}(u, w)=\mathrm{d}(u, v)+\mathrm{d}(v, w)=\delta+1$, in contradiction with the fact that the diameter of $F$ is only $\delta$.

The following can easily be derived from Lemma 4.6 and property (P1).

Corollary 4.7. Every nonempty convex subspace is of the form $\langle x, y\rangle$ for some points $x$ and $y$.

Proof. Suppose $F$ is a convex subspace of diameter $\delta$ and $x, y$ are points of $F$ at distance $\delta$ from each other. Since there exists a unique convex subspace of diameter $\delta$ through $x$ and $y$, we necessarily have $F=\langle x, y\rangle$.

Lemma 4.8. Let $F$ be a convex subspace of diameter $\delta$ and $L$ a line intersecting $F$ in a singleton $\{x\}$. Then $\langle F, L\rangle$ is a convex subspace of diameter $\delta+1$.

Proof. By Corollary 4.5, there exists a point $y \in F$ at distance $\delta$ from $x$. Let $z \in L \backslash\{x\}$. As $\mathrm{d}(z, F)=1$ and $x=\pi_{F}(z)$, we have $\mathrm{d}(z, y)=\mathrm{d}(z, x)+\mathrm{d}(x, y)=\delta+1$. Since $x$ is on a shortest path from $y$ to $z$, any convex subspace containing $y$ and $z$ contains $x$ and hence also $\langle y, x\rangle=F$. So, $\langle F, L\rangle$ necessarily coincides with $\langle y, z\rangle$ and is a convex subspace of diameter $\delta+1$.

Lemma 4.9. Let $F$ be a convex subspace of diameter $\delta$ and $Q$ a quad intersecting $F$ in a line, then $\langle F, Q\rangle$ is a convex subspace of diameter $\delta+1$.

Proof. Let $x$ be an arbitrary point of the line $L:=Q \cap F$, and let $L^{\prime}$ be a line of $Q$ through $x$ distinct from $L$. As $Q=\left\langle L, L^{\prime}\right\rangle$, we know by Lemma 4.8 that $\langle F, Q\rangle=\left\langle F, L, L^{\prime}\right\rangle=\left\langle F, L^{\prime}\right\rangle$ is a convex subspace of diameter $\delta+1$.

Definition. For every two points $x$ and $y$ of $\mathcal{S}$, let $S(x, y)$ denote the set of lines through $x$ containing a point at distance $\mathrm{d}(x, y)-1$ from $y$. Obviously, $S(x, y)=\emptyset$ if $x=y$, $S(x, y)=\{x y\}$ if $\mathrm{d}(x, y)=1$ and $S(x, y)$ is the whole set of lines through $x$ if $\mathrm{d}(x, y)=n$. 
Lemma 4.10. For every two points $x$ and $y, S(x, y)$ is the set of lines through $x$ contained in $\langle x, y\rangle$.

Proof. Put $\delta=\mathrm{d}(x, y)$. Then $F:=\langle x, y\rangle$ is a convex subspace of diameter $\delta$. Since $\widetilde{F}$ is a near polygon, any line $L$ of $F$ through $x$ contains a unique point at minimal distance $\delta-1$ from $y$, implying that $L \in S(x, y)$. Conversely, any line $K$ of $S(x, y)$ contains a point at distance $\mathrm{d}(x, y)-1$ from $y$ and the convexity of $F=\langle x, y\rangle$ then implies that $K$ must be contained in $F$.

Lemma 4.11. (1) For every convex subspace $F$ and every $x \in F$, the set of lines of $F$ through $x$ is a subspace of $\mathcal{L}_{x}$.

(2) For every two points $x$ and $y$ of $\mathcal{S}$, the set $S(x, y)$ is a subspace of $\mathcal{L}_{x}$.

Proof. (1) Let $L_{1}$ and $L_{2}$ be two distinct lines through $x$ contained in $F$. As $F$ is a convex subspace, the quad $Q=\left\langle L_{1}, L_{2}\right\rangle$ must also be contained in $F$, in particular, every line of $Q$ through $x$ is contained in $F$. This shows Claim (1).

(2) Claim (2) is a consequence of Claim (1) and Lemma 4.10.

Definition. The subspace of $\mathcal{L}_{x}$ determined by the lines of $F$ through $x$ (as in Lemma 4.11(1)) is called the subspace of $\mathcal{L}_{x}$ induced by $F$.

Lemma 4.12. Let $x_{0}, x_{1}, \ldots, x_{\delta}$ be a shortest path between two points $x_{0}$ and $x_{\delta}$. Then for every $i \in\{1,2, \ldots, \delta\}$, the subspace $S\left(x_{0}, x_{i-1}\right)$ of $\mathcal{L}_{x_{0}}$ is a proper subset of $S\left(x_{0}, x_{i}\right)$.

Proof. Let $L$ be an arbitrary line of $S\left(x_{0}, x_{i-1}\right)$. Then $L$ contains a point at distance $i-2$ from $x_{i-1}$ and thus a point at distance at most (and hence precisely) $i-1$ from $x_{i}$. So, $L$ also belongs to $S\left(x_{0}, x_{i}\right)$ implying that $S\left(x_{0}, x_{i-1}\right) \subseteq S\left(x_{0}, x_{i}\right)$. It thus suffices to show that there is some line $L \in S\left(x_{0}, x_{i}\right)$ not belonging to $S\left(x_{0}, x_{i-1}\right)$. In view of Lemma 4.10, we need to show that there is some line $L$ of $\left\langle x_{0}, x_{i}\right\rangle$ through $x_{0}$ not contained in $\left\langle x_{0}, x_{i-1}\right\rangle$. But this follows from Lemma 4.4 by extending a shortest path between $x_{i-1}$ and $x_{0}$ to a shortest path of length $i$ in $F$ between $x_{i-1}$ and $y$. Then $L=x_{0} y$ is the required line.

Lemma 4.13. Let $x$ be a point of $\mathcal{S}$. Then a subspace $S$ of $\mathcal{L}_{x}$ is induced by at most one convex subspace through $x$. If $F$ is such a convex subspace, then $F=\{x\}$ if $S=\emptyset$ and $F=\left\langle\bigcup_{L \in S} L\right\rangle$ otherwise.

Proof. If $S=\emptyset$, then $\{x\}$ is the unique convex subspace through $x$ inducing $S$. So, we may suppose that $S \neq \emptyset$.

Suppose that $S$ is induced by a convex subspace $F$ through $x$ and let $F^{\prime}$ denote the convex subspace $\left\langle\bigcup_{L \in S} L\right\rangle$. As $\bigcup_{L \in S} L$ is contained in $F$, we have $F^{\prime} \subseteq F$. By construction $F^{\prime}$ also contains all lines of $S$. Since $S$ is induced by $F$, we thus see that $S$ is also induced by $F^{\prime}$. In order to prove the uniqueness of the convex subspace through $x$ inducing $S$, it suffices to show that $F=F^{\prime}$. 
Suppose $F^{\prime}$ is properly contained in $F$. Let $\delta^{\prime}$ be the diameter of $F^{\prime}$ and $\delta$ the diameter of $F$. As there exists some line in $F$ intersecting $F^{\prime}$ in a singleton, we know by Lemma 4.8 that $\delta>\delta^{\prime}$. By Corollary 4.5, there exists a point $y$ in $F^{\prime}$ at maximal distance $\delta^{\prime}$ from $x$. By Lemma 4.4, there exists a point $z \in \Gamma_{1}(y) \cap F$ at distance $\delta^{\prime}+1$ from $x$. By Lemma 4.10 and the fact that $F^{\prime}=\langle x, y\rangle$ induces the subspace $S$ of $\mathcal{L}_{x}$, we have $S=S(x, y)$. By Lemma 4.12 and the fact that $y=\pi_{F^{\prime}}(z)$ is on a shortest path from $x \in F^{\prime}$ to $z$, we know that $S(x, y)$ is properly contained in $S(x, z)$. Since $x, z \in F$ and $F$ is a convex subspace inducing the subspace $S$ of $\mathcal{L}_{x}$, we see that $S(x, z) \subseteq S$. In summary, we have $S=S(x, y) \subsetneq S(x, z) \subseteq S$, which is clearly an impossibility. So, we indeed must have that $F^{\prime}=F$.

The following lemma is even valid in an arbitrary near polygon.

Lemma 4.14. Let $\gamma$ be a path of length $k+1$ connecting two points $x$ and $y$ at distance $k \in\{0,1, \ldots, n\}$ from each other. Then any point of $\gamma$ is contained in $\langle x, y\rangle$.

Proof. Suppose $\gamma: x=x_{0}, x_{1}, \ldots, x_{k+1}=y$ and let $l \in\{0,1, \ldots, k+1\}$ be the smallest value for which $d\left(x, x_{l}\right)<l$. Then $l \geq 2$ and $d\left(x, x_{l-1}\right)=l-1$. If $d\left(x, x_{l}\right)=l-2$, then $d\left(x_{l}, x_{k+1}\right) \geq \mathrm{d}\left(x_{0}, x_{k+1}\right)-\mathrm{d}\left(x_{0}, x_{l}\right) \geq k-l+2$, contradicting the fact that there exists a path of length $k+1-l$ between $x_{l}$ and $x_{k+1}$. Hence, $d\left(x, x_{l-1}\right)=d\left(x, x_{l}\right)=l-1$. Let $z$ be the unique point on $x_{l-1} x_{l}$ at distance $l-2$ from $x$. Then $x_{l-1} \neq z \neq x_{l}$. There is a path $\gamma_{1}$ of length $l-2$ connecting $x$ and $z$ and a path $\gamma_{2}: z, x_{l}, \ldots, x_{k+1}$ of length $k-l+2$ connecting $z$ and $x_{k+1}$. So, the concatenation of $\gamma_{1}$ and $\gamma_{2}$ is a shortest path (of length $l-2+k-l+2=k$ ) between $x$ and $y$. It follows that $z \in\langle x, y\rangle$ and $x_{i} \in\langle x, y\rangle$ for every $i \in\{l, l+1, \ldots, k+1\}$. Since $x_{l-1} \in z x_{l}$, also $x_{l-1} \in\langle x, y\rangle$. Since $x_{i}, i \in\{0,1, \ldots, l-1\}$ is contained in a shortest path from $x$ to $x_{l-1}$, also $x_{i} \in\langle x, y\rangle$ for every $i \in\{0,1, \ldots, l-1\}$. This finishes the proof that every point of $\gamma$ is contained in $\langle x, y\rangle$.

From now on, we will rely on property (P3).

Lemma 4.15. If $F$ is a convex subspace of diameter $\delta \leq n-1$, then $\widetilde{F}$ is a dual polar space of rank $\delta$.

Proof. By successively applying Lemma 4.8, we see that there exists some max $M$ through $F$. Then $F$ is a convex subspace of $\widetilde{M}$. Since $\widetilde{M}$ is a dual polar space, also $\widetilde{F}$ is a dual polar space, necessarily of rank $\delta$.

Lemma 4.16. Every local space of $\mathcal{S}$ is a (possibly degenerate) projective space of dimension at least $n-1$. All local spaces have the same dimension.

Proof. Let $x$ be an arbitrary point of $\mathcal{S}$. If $Q_{1}$ and $Q_{2}$ are two quads through $x$ which intersect in a line, then by Lemmas 4.9 and $4.15 F:=\left\langle Q_{1}, Q_{2}\right\rangle$ is a hex for which $\widetilde{F}$ is a dual polar space of rank 3. So, the lines and quads through $x$ contained in $F$ define a projective plane. Hence, every two lines of $\mathcal{L}_{x}$ that meet in a point are contained in a subgeometry of $\mathcal{L}_{x}$ that is a projective plane. This implies that $\mathcal{L}_{x}$ is a projective space. 
By Corollary 4.5, there exists a path $x=x_{0}, x_{1}, \ldots, x_{n}$ of length $n$ connecting the point $x$ with a point $x_{n}$ at maximal distance $n$ from $x$. For every $i \in\{0,1, \ldots, n\}$, let $S_{i}$ denote the subspace of $\mathcal{L}_{x}$ induced by $\left\langle x_{0}, x_{i}\right\rangle$. By Lemma 4.12, $S_{i-1}$ is properly contained in $S_{i}$ for every $i \in\{1,2, \ldots, n\}$. This implies that the dimension of the projective space $\mathcal{L}_{x}$ is at least $n-1$.

In order to show that all local spaces have the same dimension, it suffices by the connectedness of $\mathcal{S}$ to prove this for the local spaces $\mathcal{L}_{x}$ and $\mathcal{L}_{y}$, where $x$ and $y$ are distinct collinear points. But this follows from the fact that the quotient projective spaces $\mathcal{L}_{x} / x y$ and $\mathcal{L}_{y} / x y$ are isomorphic. Indeed, both $\mathcal{L}_{x} / x y$ and $\mathcal{L}_{y} / x y$ are isomorphic to the pointline geometry whose points, respectively lines, are the quads, respectively hexes, through $x y$ (natural incidence).

In the following lemma, we strengthen one of the claims of Lemma 4.13 .

Lemma 4.17. Let $x$ be a point of $\mathcal{S}$ and let $S$ be a subspace of $\mathcal{L}_{x}$ whose dimension $\delta$ is at most $n-2$. Then $S$ is induced by a necessarily unique convex subspace of diameter $\delta+1$ through $x$.

Proof. We prove this by induction on $\delta$. Obviously, the lemma holds if $\delta \in\{-1,0,1\}$. So, suppose $\delta \geq 2$ and let $S^{\prime} \subset S$ be a subspace of dimension $\delta-1$ of $\mathcal{L}_{x}$. By the induction hypothesis, $S^{\prime}$ is induced by a necessarily unique convex subspace $F^{\prime}$ of diameter $\delta$ through $x$. Let $L$ be an arbitrary line of $S \backslash S^{\prime}$ and let $F$ be the convex subspace $\left\langle F^{\prime}, L\right\rangle$ of diameter $\delta+1$ (see Lemma 4.8). Since $\delta+1 \leq n-1, \widetilde{F}$ is a dual polar space of rank $\delta+1$ by Lemma 4.15. So, the local space of $\widetilde{F}$ at the point $x$ is a projective space of dimension $\delta$, i.e., the subspace $S^{\prime \prime}$ of $\mathcal{L}_{x}$ induced by $F$ is a subspace of dimension $\delta$ of the projective space $\mathcal{L}_{x}$. Since $S^{\prime} \cup\{L\} \subseteq S^{\prime \prime} \cap S, S^{\prime}$ is a hyperplane of both $S, S^{\prime \prime}$ and $\operatorname{dim}(S)=\operatorname{dim}\left(S^{\prime \prime}\right)=\delta$ as subspaces of $\mathcal{L}_{x}$, we necessarily have $S=S^{\prime \prime}$. So, the subspace of $\mathcal{L}_{x}$ induced by $F$ coincides with $S$. The uniqueness follows from Lemma 4.13 .

Lemma 4.18. Let $x$ and $y$ be two distinct collinear points of $\mathcal{S}$. Let $S_{x}$ be a subspace of $\mathcal{L}_{x}$ containing $L=x y$ and let $\mathcal{Q}$ be the set of all quads $Q$ through $L$ that induce a line of $\mathcal{L}_{x}$ that is contained in $S_{x}$. Let $S_{y}$ denote the set of lines through $y$ that are contained in some quad of $\mathcal{Q}$. Then $S_{y}$ is a subspace of $\mathcal{L}_{y}$ having the same dimension as the subspace $S_{x}$ of $\mathcal{L}_{x}$.

Proof. Let $L_{1}$ and $L_{2}$ be two distinct lines of $S_{y}$. We need to prove that every line $L_{3}$ of $\left\langle L_{1}, L_{2}\right\rangle$ through $y$ is contained in $S_{y}$. If $L \subseteq\left\langle L_{1}, L_{2}\right\rangle$, then $\left\langle L_{1}, L_{2}\right\rangle \in \mathcal{Q}$ and hence the claim holds. We will therefore suppose that $L \nsubseteq\left\langle L_{1}, L_{2}\right\rangle$. Then $F:=\left\langle L, L_{1}, L_{2}\right\rangle$ is a hex by Lemma 4.8 and $Q_{i}:=\left\langle L, L_{i}\right\rangle \in \mathcal{Q}, i \in\{1,2\}$. By Lemma $4.15, \widetilde{F}$ is a dual polar space of rank 3 (containing the quads $Q_{1}, Q_{2}$ ) and so the subspace of $\mathcal{L}_{x}$ induced by $F$ is a projective plane. Since $S_{x}$ is a subspace of $\mathcal{L}_{x}$ and $Q_{1}, Q_{2} \in \mathcal{Q}$, every line of $F$ through $x$ must therefore belong to $S_{x}$. This implies that $\left\langle L, L_{3}\right\rangle \in \mathcal{Q}$ and hence that $L_{3} \in S_{y}$. As said before, this implies that $S_{y}$ is a subspace of $\mathcal{L}_{y}$.

In order to prove that $S_{x}$ and $S_{y}$ have the same dimension (regarded as subspaces of respectively $\mathcal{L}_{x}$ and $\mathcal{L}_{y}$ ), it suffices to show that the quotient spaces $S_{x} / x y$ and $S_{y} / x y$ 
have the same dimension. But this is obvious, since each of these quotients is isomorphic to the point-line geometry whose points are the elements of $\mathcal{Q}$ and whose lines are the hexes of $\mathcal{S}$ through $x y$ which induce a subspace of $\mathcal{L}_{x}$ contained in $S_{x}$, or equivalently, a subspace of $\mathcal{L}_{y}$ contained in $S_{y}$.

Definitions. (1) Let $x$ and $y$ be two distinct collinear points of $\mathcal{S}$, let $S_{x}$ be a subspace of $\mathcal{L}_{x}$ containing $x y$ and let $S_{y}$ be a subspace of $\mathcal{L}_{y}$ containing $x y$. If $S_{y}$ is obtained from $S_{x}$ in the way as described in Lemma 4.18 , then we say that the pair $\left(S_{x}, S_{y}\right)$ is compatible. Clearly, if $\left(S_{x}, S_{y}\right)$ is compatible, then also $\left(S_{y}, S_{x}\right)$ is compatible.

(2) Let $F$ be a max and $y \in \Gamma_{1}(F)$. Then $y$ is collinear with a unique point $x$ of $F$. Let $S_{x}^{\prime}$ be the $(n-2)$-dimensional subspace of $\mathcal{L}_{x}$ induced by $F$, let $S_{x}$ be the $(n-1)$ dimensional subspace of $\mathcal{L}_{x}$ generated by $S_{x}^{\prime}$ and $x y$, and let $S_{y}$ be the unique subspace of $\mathcal{L}_{y}$ such that $\left(S_{x}, S_{y}\right)$ is compatible. Then we say that $S_{y}$ is the subspace of $\mathcal{L}_{y}$ determined by $F$. By Lemma 4.18, the dimension of the subspace $S_{y}$ of $\mathcal{L}_{y}$ is equal to $n-1$. As $S_{x}^{\prime}$ is a hyperplane of $S_{x}$ (in $\mathcal{L}_{x}$ ), we see that the subspace $S_{y}$ of $\mathcal{L}_{y}$ consists of all lines through $y$ that are contained in a quad through $x y$ that meets $F$ in a line.

Lemma 4.19. Let $x$ be a point and $S_{x}$ a subspace of dimension $n-1$ of $\mathcal{L}_{x}$. If $F_{1}$ and $F_{2}$ are two distinct maxes through $x$ such that the set $S_{i}, i \in\{1,2\}$, of lines of $F_{i}$ through $x$ is contained in $S_{x}$, then $F_{1} \cap F_{2}$ is a convex subspace of diameter $n-2$.

Proof. By Lemma 4.16, $\mathcal{L}_{x}$ is a projective space. Since $S_{x}$ is a subspace of dimension $n-1$ and $S_{i}, i \in\{1,2\}$, is a subspace of dimension $n-2$ of $S_{x}$, we see that the dimension of $S_{1} \cap S_{2}$ is equal to $n-3$. Note that $S_{1} \neq S_{2}$ by Lemma 4.13. As $S_{1} \cap S_{2}$ is induced by the convex subspace $F_{1} \cap F_{2}$, we see that $F_{1} \cap F_{2}$ is a convex subspace of diameter $n-2$ by Lemmas 4.13 and 4.17 .

Lemma 4.20. If $F_{1}$ and $F_{2}$ are two disjoint maxes of $\mathcal{S}$ such that $F_{2} \subseteq \Gamma_{1}\left(F_{1}\right)$, then $F_{1} \subseteq \Gamma_{1}\left(F_{2}\right)$. As a consequence, $F_{1}$ and $F_{2}$ are parallel maxes at distance 1 from each other.

Proof. (a) We prove that $d\left(\pi_{F_{1}}(x), \pi_{F_{1}}(y)\right)=d(x, y)$ for any two distinct points $x$ and $y$ of $F_{2}$.

If $d\left(\pi_{F_{1}}(x), \pi_{F_{1}}(y)\right)<d(x, y)$, then there exists a path $\gamma$ of length $2+d\left(\pi_{F_{1}}(x), \pi_{F_{1}}(y)\right) \leq$ $d(x, y)+1$ connecting $x$ and $y$ and containing the points $\pi_{F_{1}}(x)$ and $\pi_{F_{1}}(y)$. Lemma 4.14 and the fact that $F_{2}$ is a convex subspace imply that all points of $\gamma$ are contained in $\langle x, y\rangle \subseteq F_{2}$, contradicting the fact that $F_{1}$ and $F_{2}$ are disjoint.

If $d(x, y)<d\left(\pi_{F_{1}}(x), \pi_{F_{1}}(y)\right)$, then there exists a path $\gamma$ of length $2+d(x, y) \leq$ $d\left(\pi_{F_{1}}(x), \pi_{F_{1}}(y)\right)+1$ connecting $\pi_{F_{1}}(x)$ and $\pi_{F_{1}}(y)$, and containing the points $x$ and $y$. Lemma 4.14 and the fact that $F_{1}$ is a convex subspace imply that all points of $\gamma$ are contained in $\left\langle\pi_{F_{1}}(x), \pi_{F_{1}}(y)\right\rangle \subseteq F_{1}$, contradicting the fact that $F_{1}$ and $F_{2}$ are disjoint.

Hence, $d\left(\pi_{F_{1}}(x), \pi_{F_{1}}(y)\right)=d(x, y)$.

(b) By (a), $F_{2}^{\prime}=\pi_{F_{1}}\left(F_{2}\right)$ is a subspace of $\mathcal{S}$ and the maximal distance between two points of $F_{2}^{\prime}$ is equal to $n-1$. Hence, $\left\langle F_{2}^{\prime}\right\rangle=F_{1}$. 
(c) We prove that $F_{2}^{\prime}$ is quad-closed. Let $x^{\prime}=\pi_{F_{1}}(x)$ and $y^{\prime}=\pi_{F_{1}}(y)$ be two points of $F_{2}^{\prime}$ at distance 2 from each other, where $x, y \in F_{2}$. We have $d(x, y)=2, d\left(x, x^{\prime}\right)=1$, $d\left(y, y^{\prime}\right)=1, d\left(x, y^{\prime}\right)=d\left(x, x^{\prime}\right)+d\left(x^{\prime}, y^{\prime}\right)=1+2=3$ and $d\left(y, x^{\prime}\right)=d\left(y, y^{\prime}\right)+d\left(y^{\prime}, x^{\prime}\right)=$ $1+2=3$. The hex $\left\langle x, y^{\prime}\right\rangle$ contains the points $x^{\prime}$ and $y$ since these points are contained on shortest paths between $x$ and $y^{\prime}$. Now, $\widetilde{\left\langle x, y^{\prime}\right\rangle}$ is a dual polar space of rank 3 (recall Lemma 4.15 and $\langle x, y\rangle \subseteq F_{1}$ and $\left\langle x^{\prime}, y^{\prime}\right\rangle \subseteq F_{2}$ are two disjoint quads of $\widetilde{\left\langle x, y^{\prime}\right\rangle}$. This implies that every point of $\left\langle x^{\prime}, y^{\prime}\right\rangle$ lies at distance 1 from some point of $\langle x, y\rangle$ and that every point of $\langle x, y\rangle$ lies at distance 1 from some point of $\left\langle x^{\prime}, y^{\prime}\right\rangle$. Hence, $\left\langle x^{\prime}, y^{\prime}\right\rangle \subseteq F_{2}^{\prime}$.

(d) By Lemma 4.15, $\widetilde{F}_{1}$ is a dual polar space of rank $n-1$. Since $F_{2}^{\prime}$ is a subspace of $F_{1}$ such that $\widetilde{F_{2}^{\prime}}$ is connected, $\left\langle F_{2}^{\prime}\right\rangle_{q}=\left\langle F_{2}^{\prime}\right\rangle$ by Lemma 3.2. Hence, $F_{2}^{\prime}=\left\langle F_{2}^{\prime}\right\rangle_{q}=\left\langle F_{2}^{\prime}\right\rangle=F_{1}$. This implies that $F_{1} \subseteq \Gamma_{1}\left(F_{2}\right)$.

Lemma 4.21. Let $F_{1}$ and $F_{2}$ be two parallel disjoint maxes at distance 1 from each other. Let $x \in F_{2}$, let $S_{x}^{\prime}$ be the subspace of $\mathcal{L}_{x}$ induced by $F_{2}$ and let $S_{x}$ be the subspace of $\mathcal{L}_{x}$ determined by $F_{1}$. Then $S_{x}^{\prime} \subseteq S_{x}$.

Proof. Put $x^{\prime}:=\pi_{F_{1}}(x)$. Let $L$ be a line of $S_{x}^{\prime}$. Let $y$ be an arbitrary point of $L \backslash\{x\}$ and put $y^{\prime}:=\pi_{F_{1}}(y)$. Since $y^{\prime}$ is contained on a shortest path from $x^{\prime}$ to $y$, the quad $\left\langle x x^{\prime}, L\right\rangle$ intersects $F_{1}$ in the line $x^{\prime} y^{\prime}$, implying that $L \in S_{x}$.

Lemma 4.22. Let $F_{1}$ be a max of $\mathcal{S}$, let $x_{2} \in \Gamma_{1}\left(F_{1}\right)$, put $x_{1}:=\pi_{F_{1}}\left(x_{2}\right)$ and let $F_{2}$ be a max through $x_{2}$ not containing $x_{1} x_{2}$ such that the subspace of $\mathcal{L}_{x_{2}}$ induced by $F_{2}$ is contained in the subspace of $\mathcal{L}_{x_{2}}$ determined by $F_{1}$. Then $F_{1}$ and $F_{2}$ are parallel maxes at distance 1 from each other.

Proof. Let $S_{i}, i \in\{1,2\}$, denote the subspace of $\mathcal{L}_{x_{i}}$ induced by $F_{i}$. After showing that $F_{1} \cap F_{2}=\emptyset$ in (a), we prove in (b) that every point of $F_{i}, i \in\{1,2\}$, at distance at most $n-2$ from $x_{i}$ lies at distance 1 from $F_{3-i}$. By relying on (b), we subsequently show in (c) that the same also holds for points of $F_{i}$ at maximal distance $n-1$ from $x_{i}$.

(a) We prove that $F_{1} \cap F_{2}=\emptyset$. Suppose $u \in F_{1} \cap F_{2}$. Then any shortest path between $x_{2} \in F_{2}$ and $u \in F_{2}$ is contained in $F_{2}$. Since $u \in F_{1}$, there exists a shortest path between $x_{2}$ and $u$ that contains the point $x_{1}=\pi_{F_{1}}\left(x_{2}\right)$. So, we would have that $x_{1} \in F_{2}$, clearly a contradiction. Hence, $F_{1} \cap F_{2}=\emptyset$.

(b) Suppose $z$ is a point of $F_{1}$ at distance $k \leq n-2$ from $x_{1}$. Put $G_{1}:=\left\langle x_{1}, z\right\rangle$ and $G:=\left\langle G_{1}, x_{1} x_{2}\right\rangle$. Then $G$ has diameter $k+1 \leq n-1$ by Lemma 4.8. Let $S_{i}^{\prime}, i \in\{1,2\}$, denote the $k$-dimensional subspace of $\mathcal{L}_{x_{i}}$ induced by $G$. Since $G \cap F_{1}=G_{1}, S_{1} \cap S_{1}^{\prime}$ is the $(k-1)$-dimensional subspace of $\mathcal{L}_{x_{1}}$ induced by $G_{1}$. By Lemma $4.15, \widetilde{G}=\left\langle G_{1}, x_{1} x_{2}\right\rangle$ is a dual polar space of rank $k+1$ and so the quads of $\widetilde{G}$ through $x_{1} x_{2}$ cover all lines of $S_{2}^{\prime}$ and intersect $G_{1}$ in lines. This implies that $S_{2}^{\prime}$ is contained in the $(n-1)$-dimensional subspace $S^{*}$ of $\mathcal{L}_{x_{2}}$ determined by $F_{1}$. By Lemma 4.21 and $x_{1} x_{2} \in S_{2}^{\prime} \backslash S_{2}, S_{2}$ is a hyperplane of $S^{*}$ not containing $S_{2}^{\prime}$. This implies that $S_{2} \cap S_{2}^{\prime}$ is a $(k-1)$-dimensional subspace of $\mathcal{L}_{x_{2}}$. By Lemma 4.17, $S_{2} \cap S_{2}^{\prime}$ is induced by a unique convex subspace $G_{2}$ of diameter $k$ through $x_{2}$. As $S_{2} \cap S_{2}^{\prime} \subseteq S_{2}$ and $S_{2} \cap S_{2}^{\prime} \subseteq S_{2}^{\prime}$, we know by Lemma 4.13 that $G_{2}$ is contained in 
$F_{2}$ and in $G$. As $\widetilde{G}$ is a dual polar space of rank $k+1$ and $G_{1}, G_{2}$ are two disjoint maxes of $\widetilde{G}$, every point of $G_{1}$ has distance 1 from $G_{2} \subseteq F_{2}$. Hence, $z \in \Gamma_{1}\left(F_{2}\right)$.

So, every point of $F_{1}$ at distance at most $n-2$ from $x_{1}$ is collinear with some point of $F_{2}$. Every line of $F_{1}$ through $x_{1}$ is thus parallel and at distance 1 from a line of $F_{2}$ through $x_{2}$. By considering suitable quads through $x_{1} x_{2}$, we thus see that the subspace of $\mathcal{L}_{x_{1}}$ induced by $F_{1}$ is contained in the subspace of $\mathcal{L}_{x_{1}}$ determined by $F_{2}$. Similarly as above, one then proves that every point of $F_{2}$ at distance at most $n-2$ from $x_{2}$ is collinear with some point of $F_{1}$.

(c) Suppose $z$ is a point of $F_{1}$ at distance $n-1$ from $x_{1}$ and let $z_{1} \in F_{1}$ denote a point collinear with $x_{1}$ at distance $n-2$ from $z$. Let $z_{2}$ denote the unique point of $F_{2}$ collinear with $z_{1}$. Then $z_{2} \sim x_{2}$. We prove that the subspace of $\mathcal{L}_{z_{2}}$ induced by $F_{2}$ is contained in the subspace of $\mathcal{L}_{z_{2}}$ determined by $F_{1}$.

So, let $L$ be an arbitrary line through $z_{2}$ contained in $F_{2}$. By (b), every point of $L$ lies in $\Gamma_{1}\left(F_{1}\right)$. So, the quad $\left\langle L, z_{1} z_{2}\right\rangle$ intersects $F_{1}$ in the line $\pi_{F_{1}}(L)$. This implies that $L$ is indeed contained in the subspace of $\mathcal{L}_{z_{2}}$ determined by $F_{1}$.

Now, by applying (b) to the tuple $\left(F_{1}, z_{2}, z_{1}, F_{2}\right)$ (instead of $\left(F_{1}, x_{2}, x_{1}, F_{2}\right)$ ), we see that every point of $F_{1}$ at distance at most $n-2$ from $z_{1}$ is contained in $\Gamma_{1}\left(F_{2}\right)$. In particular, $z \in \Gamma_{1}\left(F_{2}\right)$.

In a similar way, one proves that every point of $F_{2}$ at distance $n-1$ from $x_{2}$ is contained in $\Gamma_{1}\left(F_{1}\right)$.

(d) By (b) and (c), $F_{1} \subseteq \Gamma_{1}\left(F_{2}\right)$ and $F_{2} \subseteq \Gamma_{1}\left(F_{1}\right)$. So, $F_{1}$ and $F_{2}$ are parallel maxes at distance 1 from each other.

Definition. If $F_{1}$ and $F_{2}$ are two parallel maxes at distance 1 from each other, then $\Omega\left(F_{1}, F_{2}\right)$ denotes the set of points at distance at most 1 from $F_{1}$ and $F_{2}$.

Lemma 4.23. If $F_{1}$ and $F_{2}$ are parallel maxes at distance 1 from each other, then $\Omega\left(F_{1}, F_{2}\right)$ is a subspace.

Proof. Clearly, $\Omega\left(F_{1}, F_{2}\right)=S_{1} \cap S_{2}$, where $S_{i}, i \in\{1,2\}$, denotes the set of points at distance at most 1 from $F_{i}$. It suffices to prove that each $S_{i}, i \in\{1,2\}$, is a subspace. So, fix $i \in\{1,2\}$ and let $x$ and $y$ be two distinct collinear points of $S_{i}$. If the line $x y$ meets $F_{i}$, then the line $x y$ is contained in $S_{i}$. If the line $x y$ is disjoint from $F_{i}$, we then know that $x y \subseteq \Gamma_{1}\left(F_{i}\right)$ is parallel and at distance 1 from the line $\pi_{F_{i}}(x) \pi_{F_{i}}(y)$. So, $x y$ is contained in $\Gamma_{1}\left(F_{i}\right) \subseteq S_{i}$.

Lemma 4.24. Let $F_{1}$ and $F_{2}$ be two parallel maxes at distance 1 from each other and let $L$ be a line meeting $F_{1}$ and $F_{2}$. Then every quad through $L$ which meets $F_{1}$ in a line also meets $F_{2}$ in a line.

Proof. Put $L \cap F_{i}=\left\{x_{i}\right\}, i \in\{1,2\}$. Let $Q$ be a quad through $L$ which intersects $F_{1}$ in a line $K_{1}$. Let $y_{1} \in K_{1} \backslash\left\{x_{1}\right\}$ and let $y_{2}$ denote the unique point of $F_{2}$ collinear with $y_{1}$. Then $x_{2} \sim y_{2}$ and $y_{2}$ is contained in a shortest path from $x_{2}$ to $y_{1}$. Since $x_{2}, y_{1} \in Q$, also $y_{2} \in Q$ and hence the line $x_{2} y_{2}$ is contained in $Q$. So, $Q$ intersects $F_{2}$ in a line. 
Lemma 4.25. Let $F_{1}$ and $F_{2}$ be two parallel maxes at distance 1 from each other. Then the points of $\Omega\left(F_{1}, F_{2}\right)$ are precisely the points of $\mathcal{S}$ which are contained in some quad that intersects $F_{1}$ and $F_{2}$ in lines.

Proof. If $Q$ is a quad intersecting $F_{1}$ and $F_{2}$ in lines, then every point of $Q$ has distance at most 1 from $F_{1}$ and $F_{2}$ and hence is contained in $\Omega\left(F_{1}, F_{2}\right)$.

Conversely, suppose that $x$ is a point which has distance at most 1 from $F_{1}$ and $F_{2}$. If $x_{1}:=\pi_{F_{1}}(x)$ and $x_{2}:=\pi_{F_{2}}(x)$ are collinear, then $x$ is contained in the line $x_{1} x_{2}$ and hence also in any quad through $x_{1} x_{2}$ which intersects $F_{1}$ (and hence also $F_{2}$ ) in a line. Suppose therefore that $x_{1}$ and $x_{2}$ are not collinear. Then $\mathrm{d}\left(x_{1}, x_{2}\right)=2$. Let $x_{i}^{\prime}, i \in\{1,2\}$, denote the unique point of $F_{i}$ at distance 1 from $x_{3-i}$. As $2=\mathrm{d}\left(x_{3-i}, x_{i}\right)=\mathrm{d}\left(x_{3-i}, x_{i}^{\prime}\right)+\mathrm{d}\left(x_{i}^{\prime}, x_{i}\right)=$ $1+\mathrm{d}\left(x_{i}^{\prime}, x_{i}\right)$, the points $x_{i}^{\prime}$ and $x_{i}$ are collinear. The quad through the parallel lines $x_{1} x_{1}^{\prime}$ and $x_{2} x_{2}^{\prime}$ contains $x$ and intersects $F_{1}$ and $F_{2}$ in lines.

Lemma 4.26. Let $F_{1}$ and $F_{2}$ be two parallel maxes at distance 1 from each other and let $x \in F_{1}$. Then the set of lines through $x$ contained in $\Omega\left(F_{1}, F_{2}\right)$ is precisely the $(n-1)$ dimensional subspace $S$ of $\mathcal{L}_{x}$ determined by $F_{2}$.

Proof. Put $y:=\pi_{F_{2}}(x)$. Notice that $x y \subseteq \Omega\left(F_{1}, F_{2}\right)$ and $x y \in S$.

Suppose $L \neq x y$ is a line through $x$ contained in $\Omega\left(F_{1}, F_{2}\right)$. Then $L \subseteq \Gamma_{1}\left(F_{2}\right)$ and so $L$ projects to the line $\pi_{F_{2}}(L)$ of $F_{2}$. Since the quad $\langle L, x y\rangle$ meets $F_{2}$ in the line $\pi_{F_{2}}(L)$, we have $L \in S$.

Conversely, suppose that $L \neq x y$ belongs to $S$. Then the quad $\langle L, x y\rangle$ meets $F_{2}$ in a line implying that $L \subseteq \Gamma_{1}\left(F_{2}\right)$. Hence, $L \subseteq \Omega\left(F_{1}, F_{2}\right)$.

Lemma 4.27. Let $F_{1}$ and $F_{2}$ be two parallel maxes at distance 1 from each other. Let $x_{1} \in F_{1}$ and let $S_{x_{1}}$ be the $(n-1)$-dimensional subspace of $\mathcal{L}_{x_{1}}$ determined by $F_{2}$. If $F$ is a convex subspace of diameter at most $n-1$ through $x_{1}$ which induces a subspace of $\mathcal{L}_{x_{1}}$ that is completely contained in $S_{x_{1}}$, then $F \subseteq \Omega\left(F_{1}, F_{2}\right)$.

Proof. By Lemmas 4.13 and 4.17, the convex subspace $F$ is contained in a max which induces a subspace of $\mathcal{L}_{x_{1}}$ that is completely contained in $S_{x_{1}}$. So, it suffices to prove the lemma in the case that $F$ is a max. By Lemma 4.15 , we then know that $\widetilde{F}$ is a dual polar space.

Let $x_{2}$ be the unique point of $F_{2}$ collinear with $x_{1}$. We distinguish three cases:

(1) $F=F_{1}$. Then obviously $F \subseteq \Omega\left(F_{1}, F_{2}\right)$.

(2) $F \neq F_{1}$ and $F$ does not contain the line $x_{1} x_{2}$. Then $F \subseteq \Gamma_{1}\left(F_{2}\right)$ by Lemma 4.22 . By Lemma 4.19, $F \cap F_{1}$ is a max of the dual polar space $\widetilde{F}$ and so every point of $F$ has distance at most 1 from $F \cap F_{1}$. It follows that $F \subseteq \Omega\left(F_{1}, F_{2}\right)$.

(3) $F$ contains the line $x_{1} x_{2}$. Then $F \cap F_{i}, i \in\{1,2\}$, induces a subspace of dimension $n-3$ of $\mathcal{L}_{x_{i}}$ (as intersection of two subspaces of dimension $n-2$ in a subspace of diameter $n-1)$. By Lemmas 4.13 and 4.17, $F \cap F_{1}$ and $F \cap F_{2}$ are disjoint maxes of the dual polar space $\widetilde{F}$. It follows that every point of $F$ has distance at most 1 from $F_{1} \cap F$ and $F_{2} \cap F$. 
Lemma 4.28. Let $F_{1}$ and $F_{2}$ be two parallel maxes at distance 1 from each other. If $x \in F_{1}$ and if $y \in \Omega\left(F_{1}, F_{2}\right)$ lies at distance at most $n-1$ from $x$, then $\langle x, y\rangle \subseteq \Omega\left(F_{1}, F_{2}\right)$.

Proof. Suppose first that $y \in F_{1}$. Then $\langle x, y\rangle \subseteq F_{1} \subseteq \Omega\left(F_{1}, F_{2}\right)$.

Suppose now that $y \notin F_{1}$. Let $z$ denote the unique point of $F_{1}$ collinear with $y$ and let $S_{z}$ denote the $(n-1)$-dimensional subspace of $\mathcal{L}_{z}$ determined by $F_{2}$. Since $y \in \Omega\left(F_{1}, F_{2}\right)$, $z y \in S_{z}$ by Lemma 4.26. By Lemma 4.21, the subspace of $\mathcal{L}_{z}$ induced by $\langle x, z\rangle \subseteq F_{1}$ is contained in $S_{z}$. Since $\langle x, y\rangle$ is a dual polar space (Lemma 4.15 and $\langle x, y\rangle=\langle\langle x, z\rangle, z y\rangle$, the subspace of $\mathcal{L}_{z}$ induced by $\langle x, y\rangle$ is generated by $z y$ and the subspace of $\mathcal{L}_{z}$ induced by $\langle x, z\rangle$ and is therefore contained in $S_{z}$. By Lemma $4.27,\langle x, y\rangle \subseteq \Omega\left(F_{1}, F_{2}\right)$.

Lemma 4.29. Let $F_{1}$ and $F_{2}$ be two parallel maxes of $\mathcal{S}$ at distance 1 from each other, let $G_{1}$ be a convex subspace of diameter $i \leq n-2$ of $\widetilde{F_{1}}$ and put $G_{2}:=\pi_{F_{2}}\left(G_{1}\right)$. Then $\left\langle G_{1}, G_{2}\right\rangle$ is a convex subspace of diameter $i+1$.

Proof. Let $x_{1} \in G_{1}$ and put $x_{2}:=\pi_{F_{2}}\left(x_{1}\right) \in G_{2}$. Then $F=\left\langle G_{1}, x_{1} x_{2}\right\rangle$ is a convex subspace of diameter $i+1$ by Lemma 4.8. The map $L \mapsto \pi_{F_{2}}(L)$ defines a bijection between the set $\mathcal{A}_{1}$ of lines of $G_{1}$ through $x_{1}$ and the set $\mathcal{A}_{2}$ of lines of $G_{2}$ through $x_{2}$. Every quad through $x_{1} x_{2}$ containing a line of $\mathcal{A}_{1}$ is contained in $F$ and contains the corresponding line of $\mathcal{A}_{2}$ by Lemma 4.24. So, $F$ contains all lines of $\mathcal{A}_{2}$ and thus also the convex subspace $G_{2}$ by Lemma 4.13 . So, $F=\left\langle G_{1}, x_{1} x_{2}\right\rangle \subseteq\left\langle G_{1}, G_{2}\right\rangle \subseteq F$, implying that $\left\langle G_{1}, G_{2}\right\rangle=F$ has diameter $i+1$.

Lemma 4.30. Let $F_{1}$ and $F_{2}$ be two parallel maxes of $\mathcal{S}$ at distance 1 from each other. Let $G_{1}$ and $H_{1}$ be two disjoint maxes of $\widetilde{F_{1}}$, and put $G_{2}:=\pi_{F_{2}}\left(G_{1}\right), H_{2}:=\pi_{F_{2}}\left(G_{2}\right)$. Then $G:=\left\langle G_{1}, G_{2}\right\rangle$ and $H:=\left\langle H_{1}, H_{2}\right\rangle$ are two parallel maxes at distance 1 from each other.

Proof. By Lemma 4.29, we know that $G$ and $H$ are maxes of $\mathcal{S}$. By Lemma 4.15, we know that $\widetilde{F_{1}}, \widetilde{F_{2}}, G$ and $\widetilde{H}$ are dual polar spaces. Let $x$ be an arbitrary point of $H_{1}$ and put $y:=\pi_{G_{1}}(x), z:=\pi_{F_{2}}(x)=\pi_{H_{2}}(x)$. Let $S_{x}^{\prime}$ and $S_{x}^{\prime \prime}$ denotes the subspaces of $\mathcal{L}_{x}$ induced by respectively $H_{1}$ and $H$. Then $S_{x}^{\prime \prime}$ is the subspace of $\mathcal{L}_{x}$ generated by $S_{x}^{\prime}$ and $x z$ and does not contain $x y$. Let $S_{x}$ denote the subspace of $\mathcal{L}_{x}$ determined by $G$. As $\widetilde{F_{1}}$ is a dual polar space, the quads of $F_{1}$ through $x y$ intersect $G_{1}$ and $H_{1}$ in lines, implying that $S_{x}^{\prime} \subseteq S_{x}$. As $z=\pi_{F_{2}}(x) \in H_{2}$ is collinear with the point $\pi_{F_{2}}(y)$ of $G_{2} \subseteq G$, we see that the line $x z$ is contained in a quad through $x y$ that meets $G$ in the line $\pi_{F_{2}}(y) y$. So, also $x z \in S_{x}$. The subspace $S_{x}^{\prime \prime}$ of $\mathcal{L}_{x}$ generated by $S_{x}^{\prime}$ and $x z$ is thus contained in $S_{x}$. As $x y \notin S_{x}^{\prime \prime}$, Lemma 4.22 implies that $G$ and $H$ are two parallel maxes at distance 1 from each other.

Lemma 4.31. Let $F_{1}$ and $F_{2}$ be two parallel maxes of $\mathcal{S}$ at distance 1 from each other. Let $F$ be a max intersecting $F_{1}$ and $F_{2}$ in convex subspaces of diameter $n-2$. Then every point of $\Omega\left(F_{1}, F_{2}\right)$ has distance at most 1 from $F$.

Proof. Let $G_{i}, i \in\{1,2\}$, be the convex subspace $F_{i} \cap F$ of diameter $n-2$. Let $x \in$ $\Omega\left(F_{1}, F_{2}\right)$ and let $Q$ be a quad through $x$ intersecting $F_{1}$ in a line $L_{1}$ and $F_{2}$ in a line $L_{2}$ (recall Lemma 4.25). Then $\pi_{F_{2}}\left(G_{1}\right)=G_{2}$ and $\pi_{F_{2}}\left(L_{1}\right)=L_{2}$. We consider three cases. 
(1) $L_{1} \subseteq G_{1}$. Then $L_{1}$ and hence also $F$ contains a point at distance at most 1 from $x$.

(2) $L_{1} \cap G_{1}$ is a singleton $\{y\}$. Then $L_{2} \cap G_{2}$ is the singleton $\{z\}$, where $z=\pi_{F_{2}}(y)$. The line $y z$ is contained in the quad $Q$ and in $F$, implying that $y z$ and hence also $F$ contains a point at distance at most 1 from $x$.

(3) $L_{1} \cap G_{1}=\emptyset$. Then also $L_{2} \cap G_{2}=\emptyset$. Put $K_{1}=\pi_{G_{1}}\left(L_{1}\right)$ and $K_{2}=\pi_{F_{2}}\left(L_{2}\right)$. Let $Q_{i}, i \in\{1,2\}$, be the quad $\left\langle K_{i}, L_{i}\right\rangle$. By Lemma 4.29, $H:=\left\langle Q_{1}, Q_{2}\right\rangle=\left\langle K_{1}, L_{1}, K_{2}, L_{2}\right\rangle$ is a hex. Note that $Q=\left\langle L_{1}, L_{2}\right\rangle$ and $\left\langle K_{1}, K_{2}\right\rangle \subseteq F$ are two disjoint quads in $H$. As $\widetilde{H}$ is a dual polar space of rank 3 (Lemma 4.15), the quad $\left\langle K_{1}, K_{2}\right\rangle$ and hence also $F$ contains a point at distance 1 from $x \in Q$.

The following is an immediate consequence of Lemma 4.31.

Corollary 4.32. Let $F_{1}$ and $F_{2}$ be two parallel maxes of $\mathcal{S}$ at distance 1 from each other. Let $F_{1}^{\prime}$ and $F_{2}^{\prime}$ be two other parallel maxes of $\mathcal{S}$ at distance 1 from each other. If $F_{i} \cap F_{j}^{\prime}$ is a convex subspace of diameter $n-2$ for all $i, j \in\{1,2\}$, then $\Omega\left(F_{1}, F_{2}\right)=\Omega\left(F_{1}^{\prime}, F_{2}^{\prime}\right)$.

Lemma 4.33. Let $F_{1}$ and $F_{2}$ be two parallel maxes of $\mathcal{S}$ at distance 1 from each other and let $x$ be an arbitrary point of $\Omega\left(F_{1}, F_{2}\right)$. Then there exist two parallel maxes $F_{1}^{\prime}$ and $F_{2}^{\prime}$ of $\mathcal{S}$ at distance 1 from each other such that $x \in F_{1}^{\prime}$ and $\Omega\left(F_{1}^{\prime}, F_{2}^{\prime}\right)=\Omega\left(F_{1}, F_{2}\right)$.

Proof. Recall that $\widetilde{F_{1}}$ and $\widetilde{F_{2}}$ are dual polar spaces by Lemma 4.15 . By Lemma 4.25 , there exists a quad $Q$ containing $x$ intersecting $F_{1}$ in a line $L_{1}$ and $F_{2}$ in a line $L_{2}$. Let $G$ be a max of $\widetilde{F_{1}}$ containing $L_{1}$ and $G^{\prime}$ a max of $\widetilde{F_{1}}$ disjoint from $G$. Put $F_{1}^{\prime}:=\left\langle G, \pi_{F_{2}}(G)\right\rangle$ and $F_{2}^{\prime}:=\left\langle G^{\prime}, \pi_{F_{2}}\left(G^{\prime}\right)\right\rangle$. As $L_{1} \subseteq G$ and $L_{2}=\pi_{F_{2}}\left(L_{1}\right) \subseteq \pi_{F_{2}}(G)$, we have $Q=\left\langle L_{1}, L_{2}\right\rangle \subseteq$ $F_{1}^{\prime}$. By Lemma 4.30, $F_{1}^{\prime}$ and $F_{2}^{\prime}$ are two parallel maxes of $\mathcal{S}$ at distance 1. Moreover, $x \in Q \subseteq F_{1}^{\prime}$ and by Corollary 4.32 we know that $\Omega\left(F_{1}^{\prime}, F_{2}^{\prime}\right)=\Omega\left(F_{1}, F_{2}\right)$.

The following is an immediate corollary of Lemmas 4.26 and 4.33 .

Corollary 4.34. Let $F_{1}$ and $F_{2}$ be two parallel maxes of $\mathcal{S}$ at distance 1 from each other and let $x$ be an arbitrary point of $\Omega\left(F_{1}, F_{2}\right)$. Then the lines through $x$ contained in $\Omega\left(F_{1}, F_{2}\right)$ determine an $(n-1)$-dimensional subspace of $\mathcal{L}_{x}$.

The following is an immediate consequence of Lemmas 4.28 and 4.33 .

Corollary 4.35. Let $F_{1}$ and $F_{2}$ be two parallel maxes of $\mathcal{S}$ at distance 1 from each other. If $x, y \in \Omega\left(F_{1}, F_{2}\right)$ lie at distance at most $n-1$ from each other, then $\langle x, y\rangle \subseteq \Omega\left(F_{1}, F_{2}\right)$.

Lemma 4.36. Let $F_{1}$ and $F_{2}$ be two parallel maxes of $\mathcal{S}$ at distance 1 from each other and let $x$ and $y$ be two points of $\Omega\left(F_{1}, F_{2}\right)$. Then the distance $d^{\prime}(x, y)$ between $x$ and $y$ in the geometry $\Omega\left(\widetilde{\left.F_{1}, F_{2}\right)}\right.$ equals the distance $d(x, y)$ between $x$ and $y$ in $\mathcal{S}$. 
Proof. Note that $\mathrm{d}^{\prime}(x, y) \geq \mathrm{d}(x, y)$. By Corollary 4.35, the claim of the lemma is true if $\mathrm{d}(x, y) \leq n-1$. If $\mathrm{d}(x, y)=n$, then by considering a line $L$ through $y$ contained in $\Omega\left(F_{1}, F_{2}\right)$ and taking the unique point $z \in L$ at distance $n-1$ from $x$, we see that $\mathrm{d}^{\prime}(x, z)=n-1$ and hence that $\mathrm{d}^{\prime}(x, y)=n$.

Lemma 4.37. If $F_{1}$ and $F_{2}$ are two parallel maxes at maximal distance 1 from each other, then $\Omega\left(\widetilde{\left.F_{1}, F_{2}\right)}\right.$ is a near $2 n$-gon satisfying (P1), (P2) which is quad-closed.

Proof. Lemmas 4.23 and 4.36 imply that $\Omega\left(\widetilde{\left.F_{1}, F_{2}\right)}\right.$ is a near polygon. This near polygon is a near $2 n$-gon as the maximal distance between two points of $\Omega\left(F_{1}, F_{2}\right)$ is equal to $n$. The remaining claims follows from Corollary 4.35, which states that $\langle x, y\rangle \subseteq \Omega\left(F_{1}, F_{2}\right)$ for any two points $x$ and $y$ of $\Omega\left(F_{1}, F_{2}\right)$ at distance at most $n-1$ from each other.

Lemma 4.38. Let $F_{1}$ and $F_{2}$ be two parallel maxes of $\mathcal{S}$ at distance 1 from each other.

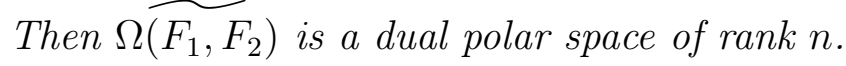

Proof. By Lemma 4.37, we know that $\left.\widehat{\Omega\left(F_{1}, F_{2}\right.}\right)$ is a near $2 n$-gon satisfying $(\mathrm{P} 1)$ and (P2). If $\Omega\left(F_{1}, F_{2}\right)$ is not a dual polar space, then there exists by Theorem 1.1 and Lemmas 4.1 . 4.3 an ovoidal point-quad pair $(x, Q)$ (in both $\left.\widehat{\Omega\left(F_{1}, F_{2}\right.}\right)$ and $\mathcal{S}$ ). Put $d(x, Q)=k$ and let $z \in Q \backslash \Gamma_{k}(x)$. Any line of $Q$ through $z$ belongs to $S(z, x)$ and is thus contained in $\langle x, z\rangle$. We thus have $\langle x, Q\rangle=\langle x, z\rangle$ and so $\langle x, Q\rangle$ is a convex subspace of diameter $k+1$ of $\mathcal{S}$. Since $x$ is ovoidal with respect to $Q, \widehat{\langle x, Q\rangle}$ is not a dual polar space. By Lemma 4.15 . $k+1=n$ or $k=n-1$. Now, let $y \in \Gamma_{n-1}(x) \cap Q$. As no line of $S(y, x)$ can be contained in $Q$, we have $\langle x, y\rangle \cap Q=\{y\}$. By Corollary 4.34, the set of lines of $\mathcal{S}$ through $y$ contained in $\Omega\left(F_{1}, F_{2}\right)$ is a subspace $S$ of dimension $n-1$ of $\mathcal{L}_{y}$. By Corollary 4.35, $\langle x, y\rangle \subseteq \Omega\left(F_{1}, F_{2}\right)$. So, the $(n-2)$-dimensional subspace $S_{1}$ of $\mathcal{L}_{y}$ induced by $\langle x, y\rangle$ is contained in $S$. The line $S_{2}$ of $\mathcal{L}_{y}$ induced by $Q$ is also contained in $S$. Since $\langle x, y\rangle \cap Q=\{y\}$, the subspaces $S_{1}$ and $S_{2}$ of $\mathcal{L}_{y}$ are disjoint. So, $S$ which contains $S_{1} \cup S_{2}$ must have dimension at least $n$, a contradiction.

Definition. Let $X$ be a subspace of $\mathcal{S}$. Then $\widetilde{X}$ is called a special subgeometry of $\mathcal{S}$ if the following properties are satisfied: (1) $\widetilde{X}$ is a dual polar space of rank $n$; (2) the distance $d\left(x_{1}, x_{2}\right)$ between two points $x_{1}$ and $x_{2}$ in the geometry $\tilde{X}$ coincides with the distance between $x_{1}$ and $x_{2}$ in the geometry $\mathcal{S}$; (3) if $x_{1}, x_{2} \in X$ lie at distance at most $n-1$ from each other, then $\left\langle x_{1}, x_{2}\right\rangle \subseteq X$. In particular, $X$ is a quad-closed set of points of $\mathcal{S}$.

The following is the main theorem of this section.

Theorem 4.39. (1) If $F_{1}$ and $F_{2}$ are parallel maxes at distance 1 from each other, then $\widehat{\Omega\left(F_{1}, F_{2}\right)}$ is a special subgeometry of $\mathcal{S}$.

(2) If $\widetilde{X}$ is a special subgeometry for some subspace $X$ of $\mathcal{S}$, then there exist two parallel maxes $F_{1}$ and $F_{2}$ at distance 1 from each other such that $X=\Omega\left(F_{1}, F_{2}\right)$. 
(3) Let $x$ and $y$ be two points of $\mathcal{S}$ at distance $n$ from each other and let $L$ be a line through $y$. Then there exists a unique special subgeometry of $\mathcal{S}$ which contains the point $x$ and the line $L$.

Proof. (1) This is an immediate consequence of Corollary 4.35 and Lemmas 4.36, 4.38.

(2) Let $F_{1}$ and $F_{2}$ be two disjoint maxes of $\widetilde{X}$. Then $F_{i}, i \in\{1,2\}$, is also a max of $\mathcal{S}$. For, if $u_{i}$ and $v_{i}$ are two points of $F_{i}$ at distance $n-1$ from each other, then $\left\langle u_{i}, v_{i}\right\rangle \subseteq X$ implies that $F_{i}=\left\langle u_{i}, v_{i}\right\rangle$. If $x \in \widetilde{X}$, then $x$ is contained in a quad of $\mathcal{S}$ meeting $F_{1}$ and $F_{2}$ in lines and hence is contained in $\Omega\left(F_{1}, F_{2}\right)$. Conversely, if $x \in \Omega\left(F_{1}, F_{2}\right)$, then Lemma 4.25 implies that $x$ is contained in a quad $Q$ which meets $F_{1}$ and $F_{2}$ in lines. The quad $Q$ is also a quad of $\widetilde{X}$ and hence $x \in X$. This proves that $X=\Omega\left(F_{1}, F_{2}\right)$.

(3) We prove that there exists at least one special subgeometry of $\mathcal{S}$ which contains the point $x$ and the line $L$. Let $z$ denote the unique point of $L$ at distance $n-1$ from $x$. Let $S_{y}$ denote the subspace of $\mathcal{L}_{y}$ determined by the $\max F_{1}:=\langle z, x\rangle$ and let $F_{2}$ denote a max through $y$ not containing $y z$ which induces a subspace of $\mathcal{L}_{y}$ contained in $S_{y}$. By Lemma 4.22, $F_{1}$ and $F_{2}$ are parallel maxes at distance 1 from each other. By (1), $\Omega\left(F_{1}, F_{2}\right)$ is a special subgeometry which satisfies the required properties.

Conversely, suppose that $X$ is a subspace of $\mathcal{S}$ such that $\{x\} \cup L \subseteq X$ and $\tilde{X}$ is a special subgeometry of $\mathcal{S}$. Since $\widetilde{X}$ is a special subgeometry of $\mathcal{S},\langle x, z\rangle \subseteq X$. By Lemma 3.1 and the fact that $X$ is a quad-closed set of $\mathcal{S}$, we have $X=\langle\langle x, z\rangle \cup L\rangle_{q}$. So, $X$ is uniquely determined and necessarily coincides with $\Omega\left(F_{1}, F_{2}\right)$.

We prove three additional structural properties of $\mathcal{S}$.

Lemma 4.40. If $\mathcal{S}$ is not a dual polar space, then for every point $x$ of $\mathcal{S}, \mathcal{L}_{x}$ is a projective space of dimension at least $2 n-1$.

Proof. We first show that all local spaces of $\mathcal{S}$ have dimension at least $n$. We subsequently use this to show that all local spaces of $\mathcal{S}$ have dimension at least $2 n-1$.

Since $\mathcal{S}$ is not a dual polar space, there exists by Theorem 1.1 and Lemmas 4.1, 4.3 an ovoidal point-quad pair $(y, Q)$. If $k:=d(y, Q)$ and $z \in Q \backslash \Gamma_{k}(y)$, then similarly as in the proof of Lemma 4.38 , we have $\langle y, Q\rangle=\langle y, z\rangle$ is a convex subspace of diameter $k+1$. Since $y$ is ovoidal with respect to $Q, \widehat{\langle y, Q\rangle}$ is not a dual polar space. By Lemma 4.15 . $k+1=n$, i.e. $k=n-1$. Now, let $z \in \Gamma_{n-1}(y) \cap Q$. Then $\langle y, z\rangle \cap Q=\{z\}$. The set $S_{1}$ of lines of $\mathcal{S}$ through $z$ contained in $\langle y, z\rangle$ is a subspace of dimension $n-2$ of $\mathcal{L}_{z}$. The set $S_{2}$ of lines of $\mathcal{S}$ through $z$ contained in $Q$ is a subspace of dimension 1 of $\mathcal{L}_{z}$. Since $\langle y, z\rangle \cap Q=\{z\}$, we have $S_{1} \cap S_{2}=\emptyset$. Hence, $\mathcal{L}_{z}$ has dimension at least $n$. By Lemma 4.16, every local space of $\mathcal{S}$ has dimension at least $n$.

Let $u$ be a point of $\mathcal{S}$ opposite to $x$, let $L_{1}$ be a line through $x$ and let $X_{1}$ denote the unique subspace of $\mathcal{S}$ containing $\{u\} \cup L_{1}$ such that $\widetilde{X_{1}}$ is a special subgeometry. Since the set of lines of $\mathcal{S}$ through $x$ contained in $X_{1}$ is a subspace of $\mathcal{L}_{x}$ of dimension $n-1$, there exists a line $L_{2}$ through $x$ not contained in $X_{1}$. Let $X_{2}$ denote the unique subspace of $\mathcal{S}$ containing $\{u\} \cup L_{2}$ such that $\widetilde{X_{2}}$ is a special subgeometry. Let $S_{i}, i \in\{1,2\}$, denote the set of lines through $x$ contained in $X_{i}$. Then $S_{i}$ is an $(n-1)$-dimensional subspace 
of $\mathcal{L}_{x}$. If $L \in S_{1} \cap S_{2}$, then $\widetilde{X_{1}}$ and $\widetilde{X_{2}}$ are two distinct special subgeometries containing $\{u\} \cup L$, in contradiction with Theorem 4.39(3). Hence, $S_{1} \cap S_{2}=\emptyset$, implying that the projective space $\mathcal{L}_{x}$ has dimension at least $2 n-1$.

Lemma 4.41. Let $\mathcal{G}$ be a special subgeometry, $x$ a point of $\mathcal{G}$ and $S_{x}$ the $(n-1)$ dimensional subspace of $\mathcal{L}_{x}$ determined by the lines through $x$ contained in $\mathcal{G}$. If $F$ is a convex subspace of diameter $\delta \in\{0,1, \ldots, n-1\}$ through $x$ such that all lines of $F$ through $x$ belong to $S_{x}$, then $F \subseteq \mathcal{G}$.

Proof. This is obviously the case if $F$ is the singleton $\{x\}$ or a line. So, suppose the diameter $\delta$ of $F$ is at least 2. By Lemma 4.15, $\widetilde{F}$ is a dual polar space of rank $\delta$ and so the subspace of $\mathcal{L}_{x}$ induced by $F$ is $(\delta-1)$-dimensional. There exist thus lines $L_{1}, L_{2}, \ldots, L_{\delta}$ through $x$ generating the subspace of $\mathcal{L}_{x}$ induced by $F$. For every $i \in\{1,2, \ldots, \delta\}$, let $F_{i}$ be the convex subspace $\left\langle L_{1}, L_{2}, \ldots, L_{i}\right\rangle$. Then $F_{i} \subseteq F$ has diameter $i$. We show by induction on $i$ that $F_{i} \subseteq \mathcal{G}$. This is clearly true if $i=1$. Suppose now that $F_{i} \subseteq \mathcal{G}$ for some $i \in\{1,2, \ldots, \delta-1\}$. Since $F_{i}$ is a convex subspace of diameter $i$, there exists by Corollary 4.5 a point $y_{i} \in F_{i}$ at distance $i$ from $x$. If $z_{i+1} \in L_{i+1} \backslash\{x\}$, then $F_{i+1}=\left\langle L_{i+1}, F_{i}\right\rangle=$ $\left\langle z_{i+1} x, x, y_{i}\right\rangle=\left\langle z_{i+1}, y_{i}\right\rangle$, where the last equality follows from the fact that $x=\pi_{F_{i}}\left(z_{i+1}\right)$ is on a shortest path between $z_{i+1}$ and $y_{i} \in F_{i}$. Since $\mathrm{d}\left(z_{i+1}, y_{i}\right)=\mathrm{d}\left(z_{i+1}, x\right)+\mathrm{d}\left(x, y_{i}\right)=$ $i+1 \leq \delta \leq n-1$, we know that $F_{i+1}=\left\langle y_{i}, z_{i+1}\right\rangle \subseteq \mathcal{G}$.

In particular, we have $F=F_{\delta} \subseteq \mathcal{G}$.

Lemma 4.42. Let $x$ be a point of $\mathcal{S}$ and $S$ a subspace of dimension $n-1$ from $\mathcal{L}_{x}$. Then there exists a unique special subgeometry $\mathcal{G}$ through $x$ such that the lines of $S$ are precisely the lines through $x$ contained in $\mathcal{G}$.

Proof. Let $S^{\prime}$ be a hyperplane of $S$ in $\mathcal{L}_{x}$ and $L \in S \backslash S^{\prime}$. By Lemma 4.17, there exists a unique max $F$ through $x$ such that the lines of $S^{\prime}$ are the lines of $F$ through $x$. By Corollary 4.5, there exists a point $y$ in $F$ at maximal distance $n-1$ from $x$. Any special geometry containing the lines of $S$ must also contain $F$ by Lemma 4.41 and hence also $L \cup\{y\}$. This in combination with Theorem 4.39(3) allows us to conclude that any special subgeometry containing the lines of $S$ must coincide with the unique special subgeometry $\mathcal{G}$ containing $L \cup\{y\}$.

Conversely, the special subgeometry $\mathcal{G}$ contains $x$ and $y$, thus also $F=\langle x, y\rangle$ and all lines of $S^{\prime}=S(x, y)$ by Lemma 4.10. The set of lines of $\mathcal{G}$ through $x$, which is an $(n-1)$ dimensional subspace of $\mathcal{L}_{x}$, therefore coincides with the subspace $S$ of $\mathcal{L}_{x}$ generated by $S^{\prime}$ and $L$.

\section{Proof of Theorem 1.2}

In this section, we suppose that $\mathcal{S}$ is a finite near $2 d$-gon, $d \geq 3$, satisfying the following two properties:

(1) Every line of $\mathcal{S}$ has at least three points and every two points at distance 2 have at least three common neighbours (in particular, $\mathcal{S}$ is dense). 
(2) If $H$ is a hex, then $\widetilde{H}$ is a dual polar space (of rank 3 ).

If not all lines of $\mathcal{S}$ are incident with the same number of points, then by [5, Theorem 1], $\mathcal{S}$ is the direct product of two near polygons $\mathcal{S}_{1}$ and $\mathcal{S}_{2}$ of diameter at least 1, i.e. the collinearity graph of $\mathcal{S}$ is the cartesian product of the collinearity graphs of $\mathcal{S}_{1}$ and $\mathcal{S}_{2}$. One can then find two points $x$ and $y$ in $\mathcal{S}$ such that $\mathrm{d}(x, y)=2$ and $\left|\Gamma_{1}(x) \cap \Gamma_{1}(y)\right|=2$, namely $x=\left(x_{1}, x_{2}\right)$ and $y=\left(y_{1}, y_{2}\right)$ where $x_{i}, y_{i}$ with $i \in\{1,2\}$ are two points of $\mathcal{S}_{i}$ for which $x_{i} \sim y_{i}$. This would however violate the claim that every two points at distance 2 have at least three common neighbours. So, there exists an $s \in \mathbb{N} \backslash\{0,1\}$ such that every line of $\mathcal{S}$ is incident with precisely $s+1$ points. By Tits' classification of polar spaces (see Section 21, we have:

Lemma 5.1. If $\mathcal{S}$ is a dual polar space, then $\mathcal{S}$ is isomorphic to $D W(2 d-1, r), D Q(2 d, r)$, $D Q^{-}(2 d+1, r), D H\left(2 d-1, r^{2}\right)$ or $D H\left(2 d, r^{2}\right)$ for some prime power $r$.

In the sequel, we suppose that $\mathcal{S}$ is not a dual polar space and derive a contradiction. We take a convex subspace $F$ of smallest possible diameter $k$ such that $\widetilde{F}$ is not a dual polar space. Then $4 \leq k \leq d$ and $\widetilde{F}$ satisfies the properties (P1), (P2), (P3) of Section 4 .

Applying Lemma 5.1 to the hexes of $\mathcal{S}$, we find:

Lemma 5.2. If $H$ is a hex, then one of the following cases occurs:

(1) $s=q$ for some prime power and $\widetilde{H} \cong D W(5, q)$ or $\widetilde{H} \cong D Q(6, q)$;

(2) $s=q^{2}$ for some prime power and $\widetilde{H} \cong D Q^{-}(7, q)$;

(3) $s=q^{\frac{1}{2}}$ for some prime power $q$ and $\widetilde{H} \cong D H(5, q)$;

(4) $s=q^{\frac{3}{2}}$ for some prime power $q$ and $\widetilde{H} \cong D H(6, q)$.

Lemma 5.3. $\widetilde{F}$ is a regular near polygon.

Proof. For any nonempty convex subspace $G$ of diameter at least 2 of $\widetilde{F}, \widetilde{G}$ is a dense near polygon and so has order $\left(s, t_{G}\right)$ for some $t_{G} \in \mathbb{N}$ by [5, Lemma 19]. In particular, $\widetilde{F}$ has order $\left(s, t_{F}\right)$. By Lemma 4.10 , it suffices to prove that $t_{G_{1}}=t_{G_{2}}$ for any two convex subspaces $G_{1}$ and $G_{2}$ of $\widetilde{F}$ of the same diameter $i \in\{2,3, \ldots, k-1\}$.

Let $Q_{j}, j \in\{1,2\}$, be a quad contained in $G_{j}$. Then $t_{G_{j}}+1=\frac{t_{Q_{j}}^{i}-1}{t_{Q_{j}}-1}$ as $\widetilde{G_{j}}$ is a dual polar space of rank $i$ (for which each local space is a projective space of dimension $i-1$ ). So, it suffices to prove the equality $t_{Q_{1}}=t_{Q_{2}}$. As $\widetilde{F}$ is connected, it suffices to prove the equality $t_{Q_{1}}=t_{Q_{2}}$ in case $Q_{1}$ and $Q_{2}$ have a common point $x$.

Let $Q_{3}$ be a quad through $x$ that intersects $Q_{1}$ and $Q_{2}$ in lines. As $H_{1}=\left\langle Q_{1}, Q_{3}\right\rangle$ and $H_{2}=\left\langle Q_{2}, Q_{3}\right\rangle$ are hexes, we indeed know by Lemma 5.2 that $t_{Q_{1}}=t_{Q_{3}}=t_{Q_{2}}$.

Let $s, t, t_{i}, i \in\{0,1, \ldots, k\}$, be the parameters of $\widetilde{F}$ regarded as a regular near polygon. Combining the above with Lemma 4.40, we find:

Lemma 5.4. We have 
- $s \in\left\{q, q^{2}, q^{\frac{1}{2}}, q^{\frac{3}{2}}\right\}$ for some prime power $q$;

- $t_{i}+1=\frac{q^{i}-1}{q-1}$ for every $i \in\{0,1, \ldots, k-1\}$;

- $t_{k}+1=\frac{q^{e}-1}{q-1}$, where $e \geq 2 k$ is some integer.

We now use the theory of distance-regular graphs and regular near polygons to derive a contradiction. Among other results, we will rely on some inequalities regarding the parameters of regular near polygons obtained by Brouwer \& Wilbrink [5], Neumaier [10] and Hiraki \& Koolen [9].

Lemma 5.5. The integer $k$ must be odd.

Proof. If $k$ is even, then the bound

$$
t_{k}+1 \leq\left(s^{2}+1\right)\left(t_{k-1}+1\right)
$$

of Brouwer and Wilbrink [5, p. 161] in combination with Lemma 5.4 implies that

$$
\frac{q^{2 k}-1}{q-1} \leq t_{k}+1 \leq\left(s^{2}+1\right)\left(t_{k-1}+1\right)=\left(s^{2}+1\right) \frac{q^{k-1}-1}{q-1}<\left(s^{2}+1\right) \frac{q^{k}-1}{q-1} .
$$

This implies that $q^{\frac{k}{2}}<s$, in contradiction with the fact that $k \geq 4$ and $s \in\left\{q, q^{2}, q^{\frac{1}{2}}, q^{\frac{3}{2}}\right\}$.

Lemma 5.6. We have $s=q^{2}$ and $t_{k}+1=\frac{q^{2 k}-1}{q-1}$.

Proof. The bound

$$
t_{k}+1 \leq \frac{s^{k}+1}{s^{k-2}+1}\left(t_{k-1}+1+s^{k-2}\right)
$$

of Neumaier [10, Theorem 3.1] in combination with Lemma 5.4 implies that

$$
\begin{gathered}
q^{2 k-1}+q^{2 k-2}<\frac{q^{2 k}-1}{q-1} \leq t_{k}+1 \leq \frac{s^{k}+1}{s^{k-2}+1}\left(t_{k-1}+1+s^{k-2}\right) \\
<s^{2}\left(\frac{q^{k-1}-1}{q-1}+s^{k-2}\right)<s^{2}\left(q^{k-1}+s^{k-2}\right) .
\end{gathered}
$$

If $s \in\left\{q, q^{\frac{1}{2}}, q^{\frac{3}{2}}\right\}$, then this implies that $q^{2 k-1}+q^{2 k-2}<q^{k+2}+q^{\frac{3 k}{2}}$, in contradiction with $k+2<2 k-1$ and $\frac{3 k}{2} \leq 2 k-2$. Hence, $s=q^{2}$.

If $t_{k}+1 \neq \frac{q^{2 k}-1}{q-1}$, then the above-mentioned Neumaier bound in combination with Lemma 5.4 implies that

$$
\begin{aligned}
q^{2 k}+q^{2 k-1}+\cdots+q+1 & \leq t_{k}+1 \leq \frac{s^{k}+1}{s^{k-2}+1}\left(t_{k-1}+1+s^{k-2}\right)<s^{2}\left(t_{k-1}+1+s^{k-2}\right) \\
& \leq q^{4}\left(1+q+\cdots+q^{k-2}+q^{2 k-4}\right)
\end{aligned}
$$

clearly a contradiction. Hence, $t_{k}+1=\frac{q^{2 k}-1}{q-1}$. 
Lemma 5.7. We have $k \in\{5,7\}$. If $k=7$, then $q \in\{2,3,4\}$.

Proof. Put $\tau:=\frac{2 k+1}{2 k-2}$. Then the inequality

$$
\frac{t_{k}+1}{t_{k-1}+1}<s\left(2 s^{\tau}+\frac{1}{2}\right)^{2}+1
$$

of Hiraki and Koolen [9, Theorem 1] in combination with Lemmas 5.4 and 5.6 implies that

$$
q^{k+1}+1<\frac{q^{2 k}-1}{q^{k-1}-1}=\frac{t_{k}+1}{t_{k-1}+1}<s\left(2 s^{\tau}+\frac{1}{2}\right)^{2}+1=q^{2}\left(2 q^{2 \tau}+\frac{1}{2}\right)^{2}+1,
$$

or $q^{\frac{k-1}{2}}-2 q^{2 \tau}-\frac{1}{2}<0$. If $k \geq 9$, then $q^{\frac{k-1}{2}}-2 q^{2 \tau}-\frac{1}{2} \geq q^{4}-2 q^{\frac{19}{8}}-\frac{1}{2}=q^{\frac{19}{8}}\left(q^{\frac{13}{8}}-2\right)-\frac{1}{2}>0$, a contradiction. Hence, $k \in\{5,7\}$ by Lemma 5.5. If $k=7$, then $q^{3}-2 q^{\frac{5}{2}}-\frac{1}{2}<0$ implies that $q \in\{2,3,4\}$.

By Lemmas 5.4, 5.6 and 5.7, the following possibilities for the parameters remain:

(1) $k=5, t_{2}=q, s=q^{2}, t_{3}=q^{2}+q, t_{4}=q^{3}+q^{2}+q$ and $t_{5}=q^{9}+q^{8}+q^{7}+q^{6}+q^{5}+$ $q^{4}+q^{3}+q^{2}+q$,

(2) $k=7, t_{2}=q, s=q^{2}, t_{3}=q^{2}+q, t_{4}=q^{3}+q^{2}+q, t_{5}=q^{4}+q^{3}+q^{2}+q$, $t_{6}=q^{5}+q^{4}+q^{3}+q^{2}+q$ and $t_{7}=q^{13}+q^{12}+q^{11}+q^{10}+q^{9}+q^{8}+q^{7}+q^{6}+q^{5}+q^{4}+q^{3}+q^{2}+q$, where $q \in\{2,3,4\}$.

The collinearity graph $\Gamma$ of $\widetilde{F}$ is a distance regular graph of diameter $k$, whose parameters are $a_{i}=(s-1)\left(t_{i}+1\right), b_{i}=s\left(t_{k}-t_{i}\right), c_{i}=t_{i}+1$ for $i \in\{0,1, \ldots, k\}$. Now, define polynomials $u_{i}(x) \in \mathbb{Q}(x)$ in the following recursive way:

$u_{0}(x):=1, u_{1}(x):=\frac{x}{b_{0}}, u_{i+1}(x):=\frac{1}{b_{i}}\left(-c_{i} \cdot u_{i-1}(x)+\left(x-a_{i}\right) \cdot u_{i}(x)\right), i \in\{1,2, \ldots, k-1\}$.

If $\theta$ is an eigenvalue of $\Gamma$, then $\left(u_{0}(\theta), u_{1}(\theta), \ldots, u_{k}(\theta)\right)$ is called the standard sequence of $\Gamma$ corresponding to $\theta$ ([4, Section 4.1]).

Now, the smallest eigenvalue of $\Gamma$ is equal to $-\left(t_{k}+1\right)$, see Brouwer and Wilbrink [5, p. 160-161]. One easily verifies that the standard sequence of this eigenvalue is equal to $\left(1,-\frac{1}{s}, \frac{1}{s^{2}}, \ldots,\left(-\frac{1}{s}\right)^{k}\right)$. If $n_{i}, i \in\{0,1, \ldots, k\}$, denotes the constant number of points of $F$ at distance $i$ from a given point of $F$, then

$$
n_{i}=\frac{s^{i} \cdot \prod_{j=0}^{i-1}\left(t-t_{j}\right)}{\prod_{j=1}^{i}\left(t_{j}+1\right)}
$$

by standard counting (see [5, 7]). By Biggs [1, p. 19], [2, p. 95] (see also [4, Theorem 4.1.4]), the multiplicity of an eigenvalue $\theta$ of $\Gamma$ is equal to

$$
\frac{|F|}{\sum_{i=0}^{k} n_{i} \cdot\left(u_{i}(\theta)\right)^{2}}=\frac{\sum_{i=0}^{k} n_{i}}{\sum_{i=0}^{k} n_{i} \cdot\left(u_{i}(\theta)\right)^{2}} .
$$


In particular, the multiplicity of the eigenvalue $-\left(t_{k}+1\right)$ is equal to

$$
m:=\left(\sum_{i=0}^{k} n_{i}\right)\left(\sum_{i=0}^{k} n_{i} s^{-2 i}\right)^{-1} .
$$

(1) Now, consider the case where $k=5, t_{2}=q, s=q^{2}, t_{3}=q^{2}+q, t_{4}=q^{3}+q^{2}+q$ and $t_{5}=q^{9}+q^{8}+q^{7}+q^{6}+q^{5}+q^{4}+q^{3}+q^{2}+q$. Then one calculates that

$$
m=f_{1}(q)+\frac{f_{2}(q)}{f_{3}(q)}
$$

where

$$
\begin{aligned}
f_{1}(q)= & q^{18}-q^{14}+3 q^{10}-q^{9}-q^{8}-4 q^{6}+3 q^{5}+4 q^{4}-2 q^{3}+3 q^{2}-6 q-7, \\
f_{2}(q)= & 9 q^{22}+10 q^{21}+29 q^{20}+44 q^{19}+53 q^{18}+73 q^{17}+105 q^{16}+127 q^{15} \\
& +161 q^{14}+178 q^{13}+178 q^{12}+179 q^{11}+188 q^{10}+183 q^{9}+178 q^{8} \\
& +164 q^{7}+120 q^{6}+86 q^{5}+80 q^{4}+59 q^{3}+46 q^{2}+40 q+14 \\
f_{3}(q)= & q^{23}+q^{22}+2 q^{21}+3 q^{20}+6 q^{19}+7 q^{18}+10 q^{17}+12 q^{16}+15 q^{15} \\
& +17 q^{14}+20 q^{13}+20 q^{12}+21 q^{11}+20 q^{10}+19 q^{9}+18 q^{8}+17 q^{7} \\
& +13 q^{6}+10 q^{5}+8 q^{4}+6 q^{3}+4 q^{2}+4 q+2 .
\end{aligned}
$$

Notice that all the nonzero coefficients of the polynomials $f_{2}(q)$ and $f_{3}(q)$ are positive. If $C_{i}, i \in\{0,1, \ldots, 22\}$, denotes the coefficient of $q^{i}$ in the polynomial $f_{2}(q)$ and $D_{i}$, $i \in\{0,1, \ldots, 23\}$, denotes the coefficient of $q^{i}$ in the polynomial $f_{3}(q)$, then we have that $C_{i} \leq \frac{44}{3} D_{i+1}$ for every $i \in\{0,1, \ldots, 22\}$. This implies that $0<f_{2}(q)<f_{3}(q)$ for every $q \geq 15$. So, $m$ cannot be integral if $q \geq 15$. If $q<15$, i.e. $q \in\{2,3,4,5,7,8,9,11,13\}$, then one can also easily verify that $\frac{f_{2}(q)}{f_{3}(q)}$ and hence also $m$ is not integral. So, the case $k=5$ cannot occur.

(2) Consider now the case $k=7, t_{2}=q, s=q^{2}, t_{3}=q^{2}+q, t_{4}=q^{3}+q^{2}+q, t_{5}=q^{4}+q^{3}+$ $q^{2}+q, t_{6}=q^{5}+q^{4}+q^{3}+q^{2}+q, t_{7}=q^{13}+q^{12}+q^{11}+q^{10}+q^{9}+q^{8}+q^{7}+q^{6}+q^{5}+q^{4}+q^{3}+q^{2}+q$, where $q \in\{2,3,4\}$. Putting $m_{q}=m$, we find

$$
\begin{aligned}
& m_{2}=66144536+\frac{105089758162492}{145959280562129} \notin \mathbb{N}, \\
& m_{3}=2538429414694+\frac{2340656569502919078170245}{2885075998038235811542463} \notin \mathbb{N}, \\
& m_{4}=4502504861592725+\frac{2932739491798122360242337990887}{2946142492535696835096707829397} \notin \mathbb{N} .
\end{aligned}
$$

None of these values is thus integral, and so this case cannot occur. We thus conclude that $\mathcal{S}$ must be a dual polar space. 


\section{Acknowledgment}

The author wants to express his gratitude to Hiroshi Suzuki for communicating his desire to him to have an alternative proof of Theorem 1.2 that does not rely on Tits' strong results on covering of chamber systems. The author also wants to thank him for his comments on an earlier draft.

\section{References}

[1] N. Biggs. Intersection matrices for linear graphs. pp. 15-23 in Combinatorial Mathematics and its Applications (Proc. Conf., Oxford, 1969). Academic Press, 1971.

[2] N. Biggs. Finite groups of automorphisms. London Mathematical Society Lecture Note Series 6. Cambridge University Press, 1971.

[3] A. E. Brouwer and A. M. Cohen. Local recognition of Tits geometries of classical type. Geom. Dedicata 20 (1986), 181-199.

[4] A. E. Brouwer, A. M. Cohen and A. Neumaier. Distance-regular graphs. Ergebnisse der Mathematik und ihrer Grenzgebiete (3) 18. Springer-Verlag, 1989.

[5] A. E. Brouwer and H. A. Wilbrink. The structure of near polygons with quads. Geom. Dedicata 14 (1983), 145-176.

[6] P. J. Cameron. Dual polar spaces. Geom. Dedicata 12 (1982), 75-85.

[7] B. De Bruyn. Near polygons. Birkhäuser, 2006.

[8] B. De Bruyn. An introduction to incidence geometry. Birkhäuser, 2016.

[9] A. Hiraki and J. Koolen. A generalization of an inequality of Brouwer-Wilbrink. J. Combin. Theory Ser. A 109 (2005), 181-188.

[10] A. Neumaier. Krein conditions and near polygons. J. Combin. Theory Ser. A 54 (1990), 201-209.

[11] S. E. Payne and J. A. Thas. Finite generalized quadrangles. Second edition. EMS Series of Lectures in Mathematics. European Mathematical Society, 2009.

[12] S. Shad and E. Shult. The near $n$-gon geometries. Unpublished manuscript.

[13] E. Shult and A. Yanushka. Near $n$-gons and line systems. Geom. Dedicata 9 (1980), $1-72$.

[14] J. Tits. Buildings of spherical type and finite BN-pairs. Lecture Notes in Mathematics 386. Springer-Verlag, 1974.

[15] J. Tits. A local approach to buildings. pp. 519-547 in The geometric vein. Springer, 1981. 